\title{
Astrocytes propel neurovascular dysfunction during cerebral cavernous malformation lesion formation
}

\author{
Miguel Alejandro Lopez-Ramirez, ${ }^{1,2}$ Catherine Chinhchu Lai, ${ }^{1}$ Shady Ibrahim Soliman, ${ }^{1}$ Preston Hale, ${ }^{1}$ Angela Pham, ${ }^{1}$ Esau J. Estrada, ${ }^{1}$ \\ Sara McCurdy, ${ }^{1}$ Romuald Girard, ${ }^{3}$ Riya Verma, ${ }^{1}$ Thomas Moore, ${ }^{3}$ Rhonda Lightle, ${ }^{3}$ Nicholas Hobson, ${ }^{3}$ Robert Shenkar, ${ }^{3}$ Orit Poulsen, ${ }^{4}$ \\ Gabriel G. Haddad, ${ }^{4,5,6}$ Richard Daneman, ${ }^{2}$ Brendan Gongol, ${ }^{1}$ Hao Sun, ${ }^{1}$ Frederic Lagarrigue, ${ }^{1}$ Issam A. Awad, ${ }^{3}$ and Mark H. Ginsberg ${ }^{1}$ \\ 'Department of Medicine, and ²Department of Pharmacology, University of California, San Diego, La Jolla, California, USA. ${ }^{3}$ Neurovascular Surgery Program, Department of Neurological Surgery, The \\ University of Chicago Medicine and Biological Sciences, Chicago, Illinois, USA. ${ }^{4}$ Department of Pediatrics, and ${ }^{5}$ Department of Neuroscience, Division of Respiratory Medicine, University of California, San \\ Diego, La Jolla, California, USA. ${ }^{6}$ Rady Children's Hospital, San Diego, California, USA.
}

\begin{abstract}
Cerebral cavernous malformations (CCMs) are common neurovascular lesions caused by loss-of-function mutations in 1 of 3 genes, including KRIT1 (CCM1), CCM2, and PDCD10 (CCM3), and generally regarded as an endothelial cell-autonomous disease. Here we reported that proliferative astrocytes played a critical role in CCM pathogenesis by serving as a major source of VEGF during CCM lesion formation. An increase in astrocyte VECF synthesis is driven by endothelial nitric oxide (NO) generated as a consequence of KLF2- and KLF4-dependent elevation of eNOS in CCM endothelium. The increased brain endothelial production of NO stabilized HIF-1 $\alpha$ in astrocytes, resulting in increased VECF production and expression of a "hypoxic" program under normoxic conditions. We showed that the upregulation of cyclooxygenase-2 (COX-2), a direct HIF- $1 \alpha$ target gene and a known component of the hypoxic program, contributed to the development of CCM lesions because the administration of a COX-2 inhibitor significantly prevented the progression of CCM lesions. Thus, non-cell-autonomous crosstalk between CCM endothelium and astrocytes propels vascular lesion development, and components of the hypoxic program represent potential therapeutic targets for CCMs.
\end{abstract}

\section{Introduction}

Cerebral cavernous malformations (CCMs) are caused by gross brain endothelial changes that lead to blood-brain barrier (BBB) dysfunction, resulting in significant morbidity and mortality $(1,2)$. CCMs affect approximately 1 in 200 children and adults, causing a lifelong risk of chronic and acute hemorrhage and consequent disabilities from neurological deficits, for which there is no current effective pharmacologic therapy (3-6). Inherited germline and somatic loss of function mutations in the genes KRIT1 (Krev1 interaction trapped gene 1, CCM1), CCM2 (Malcavernin), PDCD10 (programmed cell death protein 10, CCM3) are associated with CCMs (5, 7-9). Notably, CCMs have been recognized as an endothelial cell-autonomous disease because endothelial-specific inactivation of murine Krit1, Ccm2, or Pdcd10, results in brain and retinal vascular lesions similar to those in patients with CCM (10-13). These murine studies are also supported by the finding of a "second hit" on the normal KRIT1 or PDCD10 allele in the CCM endothelium $(14,15)$. Recent studies in CCM animal models proposed an endothelial cell nonautonomous mechanism of lesion formation in the CNS (16-19). However, there has been to date no compelling mechanistic explanation for the propensity of CCM lesions to form in the central nervous system (CNS).

Authorship note: $\mathrm{CCL}, \mathrm{SIS}, \mathrm{PH}, \mathrm{HS}, \mathrm{FL}$, and IAA contributed equally to this work Conflict of interest: The authors have declared that no conflict of interest exists. Copyright: @ 2021, American Society for Clinical Investigation.

Submitted: April 27, 2020; Accepted: May 24, 2021; Published: July 1, 2021.

Reference information: J Clin Invest. 2021;131(13):e139570.

https://doi.org/10.1172/JCl139570.
Recent studies have shown that loss of CCM genes in neuroglia or stromal cells may contribute to CCM pathogenesis through non-cell-autonomous mechanisms $(18,20,21)$. Although CCM is a disease that affects the neurovascular unit, there is a limited understanding of the crosstalk between the CCM endothelium and astrocytes $(20,22,23)$. Astrocytes are the most abundant cell in the CNS and form part of the neurovascular unit that maintains a functional BBB (24-26). Additionally, astrocytes respond to multiple forms of insults and diseases by a process called reactive astrogliosis or astrocytosis, which involves changes in morphology, function, and gene expression $(27,28)$. Reactive astrogliosis increases secretion of VEGF and can contribute to the development and progression of CNS disease (29-31). Previous studies have demonstrated elevated levels of angiogenic factor VEGF in CCM lesions, and in the plasma of individuals with the hereditary and sporadic form of the disease (32-35). VEGF signaling mediates disruption of the brain endothelial barrier by disassembly of inter-endothelial junctions (36) and can cause hemorrhages (37, 38 ), both prominent features of CCM lesions (2, 6, 10, 39, 40). We and others found that CCM led to a dramatic increase in VEGF signaling and that blocking VEGF signaling prevents subsequent formation of CCMs $(6,41)$. However, despite these emerging studies, the mechanism and source of VEGF production in CCM lesions in vivo remain elusive.

Endothelial nitric oxide synthase (eNOS, the product of the NOS3 gene) is an enzyme that generates nitric oxide (NO) and it is responsible for vascular remodeling, angiogenesis and vascular tone $(42,43)$. Previous studies indicate that eNOS con- 
A

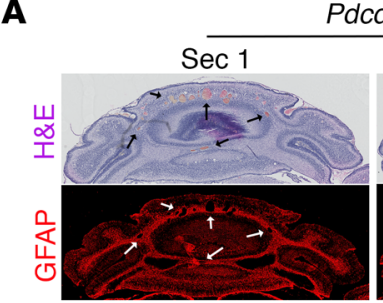

B
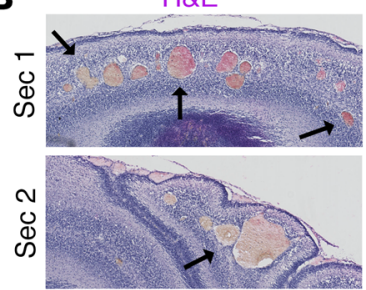

$\operatorname{Pdcd} 10^{\mathrm{ECKO}}$

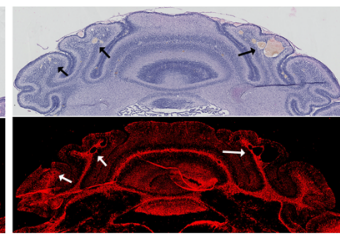

GFAP
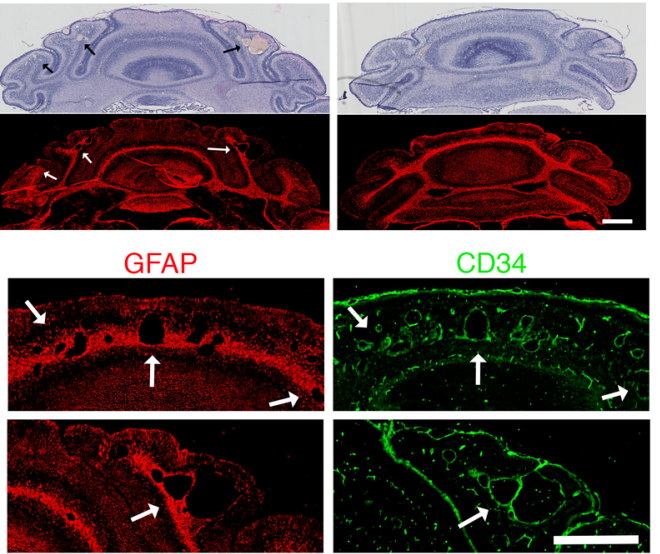

CD34

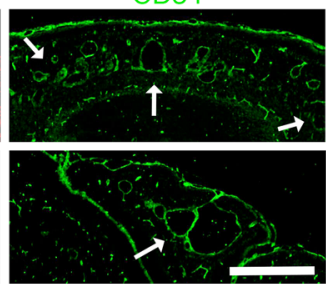

C

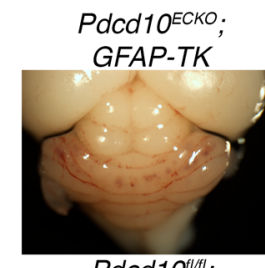

Pdcd10 $0^{\text {Iffit; }}$ GFAP-TK

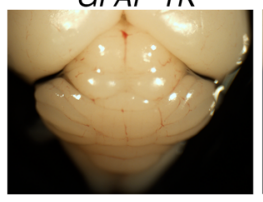

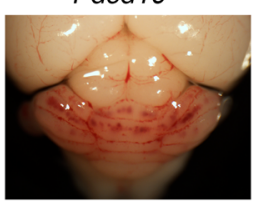

$\operatorname{Pdcd} 10^{\text {flft }}$

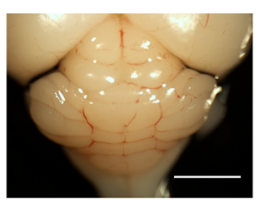

Pdcd10 ECKO
D

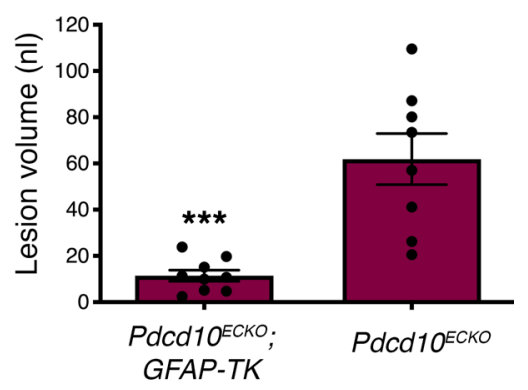

Figure 1. Astrocytes contribute to cerebral cavernous malformation development. (A) Histological analysis of cerebellar sections from P9 Pdcd10 ${ }^{\text {ECKO }}$ and littermate control Pdcd10 ${ }^{f l / f l}$ mice. Low magnification of CCM lesions detected in sections stained by hematoxylin and eosin. CCM lesions spatially developed on fibrous astrocyte areas positive for GFAP immunostaining (red). Arrows indicate CCM lesions. (B) Magnified region from sections 1 and 2 (Sec1 and $\mathrm{Sec} 2$ ) from $P d c d 10^{E C K O}$ mice in (A). Dramatic vascular dilation is shown with immunohistochemistry for the endothelial marker CD34 (green). Arrows indicate CCM lesions. (C) Administration of $1.5 \mathrm{mg} / \mathrm{kg}$ GCV in neonatal mice at $\mathrm{P7}$ markedly reduced vascular lesions in $\mathrm{Pg} P d c d 10^{E C K O}$ GFAP-TK hindbrains when compared with GCV-treated littermate $P d c d 10^{E C K O}$ controls. (D) Quantification of lesion volumes by micro-CT analysis from mice in experiments showed in (C); $n=8$ or 9 mice in each group. Data are mean \pm SEM. ${ }^{* *} P<$ 0.001 , as determined by Student's $t$ test. Scale bars: $500 \mu \mathrm{m}$ (A and B); 2 mm (C). tributes to VEGF-induced angiogenesis and vascular permeability $(44)$ through NO production $(45,46)$. Here we show that astrocytes are a major source of VEGF during CCM pathogenesis and that depletion of proliferative astrocytes prevents CCM lesion development. We show that CCM endothelium-induced elevation of astrocyte-derived VEGF in neonatal and juvenile brains occurs through elevation of brain eNOS/NO-dependent signaling. Production of NO in CCM endothelium contributes to normoxic stability of HIF- $1 \alpha$ in astrocytes. Our findings indicate that the eNOS increase in human CCM endothelium is ascribable to the elevation of the transcription factors KLF2 and KLF4, previously implicated in the CCM pathogenesis (6, $13,40,47,48)$. Furthermore, genetic inactivation of one copy of the Nos3 gene is sufficient to attenuate CCM endothelial NO production, HIF-1 $\alpha$ protein stability and astrocyte-derived VEGF expression. Our results further reveal that CCM tissue results in increased HIF activity under normoxic conditions. We show that pharmacological inhibition of a HIF- $1 \alpha$ regulated gene, cyclooxygenase-2, ameliorates the development of CCM lesions in animal models. Overall, these findings indicate that a non-cell-autonomous mechanism mediated by CCM endothelium-driven normoxic stabilization of HIF- $1 \alpha$ and increase of VEGF in astrocytes makes a major contribution to CCM pathogenesis. Understanding the crosstalk between dysfunctional brain vasculature and components of the neurovascular unit (e.g., astrocytes) has the potential to lead to the development of novel therapeutic strategies for CCM disease as exemplified by our finding that inhibition of HIF1-driven COX-2 by an approved and a well-tolerated drug can ameliorate murine models of CCM disease.

\section{Results}

Astrocytes contribute to cerebral cavernous malformations development. CCMs have been studied as an endothelial cell-autonomous disease $(6,14,15)$, marked by changes in the CNS vasculature due to the loss of endothelial PDCD1O, KRIT1, or CCM2 (10-12). Although it is a disease that affects the neurovascular unit, we know little about whether astrocytes influence CCM pathogenesis (20, 22). This prompted us to investigate the relationship between astrocytes and CCM lesion development in murine CCMs (Figure 1, A and B, and Supplemental Figure 1; supplemental material available online with this article; https://doi.org/10.1172/JCI139570DS1). Neonatal endo-

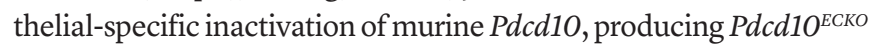
mice (40), results in cerebellar vascular lesions detected in sections stained by hematoxylin and eosin (Figure 1, A and B, and Supplemental Figure 1) or by observing dramatic vascular dilation by staining the endothelial marker CD34 at P9 (Figure 1, A and B). CCM lesions have high propensity to develop in GFAP-positive fibrous

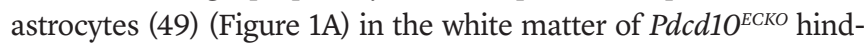

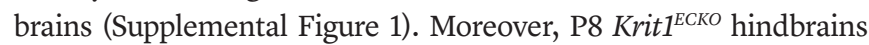
show the same spatial distribution of CCM lesions among astrocytes positive for GFAP on the white matter tract (Supplemental Figure 1). To examine the role of astrocytes, we generated Pdcd1O ECKO GFAPTK mice (Pdgfb-iCreERT2 Pdcd1 ${ }^{f / f l}$ GFAP-TK [thymidine kinase] 
A

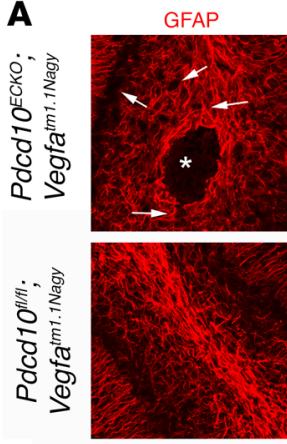

B

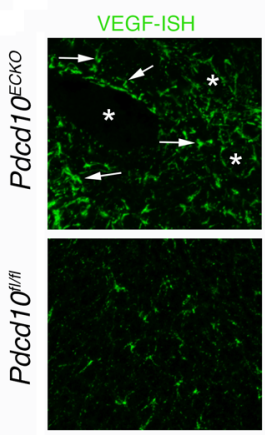

C
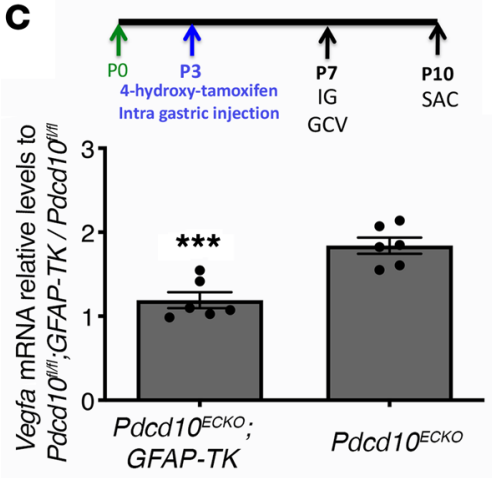

b-Gal-VEGF
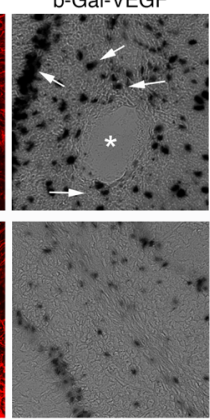
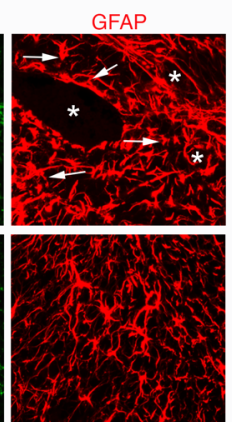

\section{D}
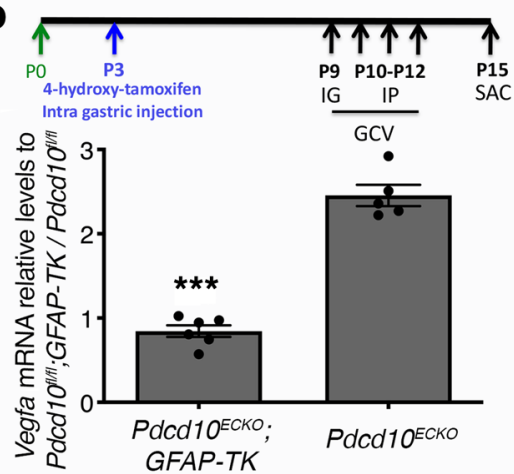

Figure 2. Astrocyte-derived VEGF increased during cerebral cavernous malformation development. (A) Confocal microscopy of cerebellar cortex from P10 Pdcd10 ECKO and littermate control Pdcd10f//fl mice stained for GFAP-positive astrocytes (red), $\beta$-gal/VEGF expression detected by $X$-gal staining (black), and endothelial marker isolectin B4 (green). Asterisk indicates vascular lumen of CCM lesion. Arrows indicate $\beta$-gal/VEGF in GFAP-positive astrocytes $(n=4)$. (B) ISH for VEGF (green) combined with immunohistochemistry to identify GFAP-positive astrocytes (red), endothelial marker isolectin B4 (IB4; white). Asterisk indicates vascular lumen of CCM lesion. Arrows indicate VEGF and GFAP-positive astrocyte colocalization $(n=2)$. (C) Quantification of Vegfa mRNA levels in P10 Pdcd10 EСKO GFAP-TK and Pdcd10ECKO hindbrains when compared with littermate Pdcd10 ${ }^{f / / f l}$ GFAP-TK or Pdcd10 fl/fl controls, respectively, as assessed by RT-qPCR. All mice received IG administration of $1.5 \mathrm{mg} / \mathrm{kg}$ GCV in neonatal at P7 (SEM, $n=6$ mice in each group). (D) Quantification of Vegfa mRNA levels in P15 Pdcd10 ECKO GFAP-TK and Pdcd10 ECKO hindbrains when compared with littermate $P d c d 10^{f / f l}$ GFAP-TK or Pdcd10 fl/fl controls, respectively, as assessed by RT-qPCR. All mice received IG administration of 1.5 $\mathrm{mg} / \mathrm{kg}$ CCV in neonatal at $P 9$ and 3 consecutive doses of IP $1.5 \mathrm{mg} / \mathrm{kg}$ GCV at P10 to P12 (SEM, $n=5$ or 6 mice in each group). (E) Prominent lesions are present in the cerebellum of P15 GCV-treated Pdcd10 ${ }^{E C K O}$ mice, whereas extensive reduction in lesions are observed in GCV-treated Pdcd10ECKO GFAP-TK littermate mice ( $n=6$ or 7). Data are mean $\pm \mathrm{SEM}$. ${ }^{* *} P<0.001$ (comparison to Pdcd10 ECKO littermates); determine by Student's $t$ test. Scale bars: $100 \mu \mathrm{m}$ (A and B).

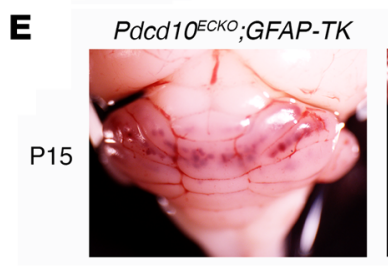

$\mathbf{E}$ mice) in which proliferative astrocytes are selectively depleted in a time-controlled manner, by administration of antiviral agent ganciclovir (GCV) $(50,51)$. Administration of $20 \mathrm{mg} / \mathrm{kg}$ GCV to neonatal mice at P7 markedly reduced hindbrain vascular lesions in P9 Pdcd10 ECKO GFAP-TK mice when compared with GCV-treated littermate $P d c d 10^{E C K O}$ controls (Figure $1 \mathrm{C}$ ). To quantify CCM formation, we imaged $\mathrm{P} 9$ hindbrains using contrast-enhanced, high-resolution $\mathrm{x}$-ray microcomputed tomography (micro-CT), and measured lesion volumes using semiautomated software (52). Blinded measurement of total CCM lesion volume showed that GCV-treated Pdcd10 ECKO GFAP-TK mouse hindbrains exhibited significant reduction of CCM lesions compared with GCV-treated $P d c d 1 O^{E C K O}$ mouse hindbrains littermates (Figure 1D). These data suggest that in CCM disease, proliferative astrocytes participate in vascular lesion formation.
Astrocyte-derived VEGF is increased during CCM development. CCMs exhibit increased VEGF signaling $(6,33,34)$ due to the loss of an anti-angiogenic checkpoint (6). To ascertain a potential source of increased expression of VEGF, we introduced a VEGF reporter, Vegf $a^{\text {tm1.1Nagy }}$, in CCM animal models. Veg $\mathrm{fa}^{\text {tm1.1Nagy }}$ carries a nuclear-localized beta-galactosidase ( $\beta$-gal) knock-in at the 3' UTR of the Vegfa gene locus that permits single-cell monitoring of VEGF expression (53). We observed an increase in $\beta$-gal/ VEGF expression, as shown by X-gal staining, in areas surrounding CCM lesions in P10 Pdcd10 ECKO Vegfat ${ }^{\text {tm1.1Nagy }}$ hindbrains when compared with $P d c d 10^{f / f l} V e g f a^{\text {tm1.1Nagy }}$ littermate controls (Figure $2 \mathrm{~A})$. We found that most $\beta$-gal/VEGF ${ }^{+}$cells colocalized with the GFAP marker of fibrous astrocytes and Bergman glia located within Purkinje cell layer (49) in Pdcd10 ECKO Vegfa $a^{\text {tm1.1Nagy }}$ (Figure 2A). 
A
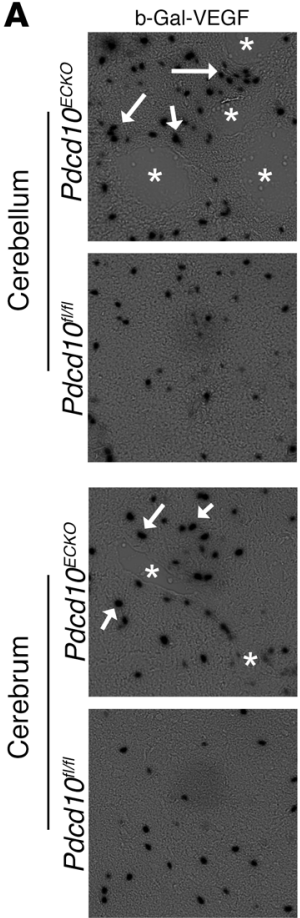

B

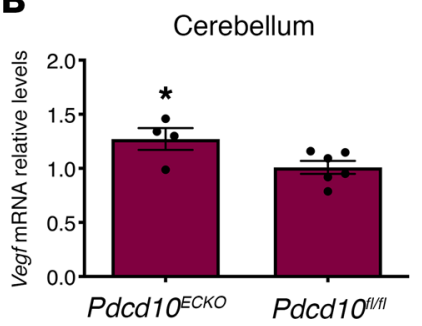

GFAP
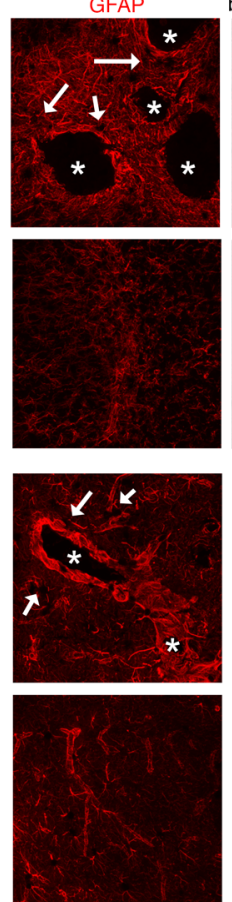

C

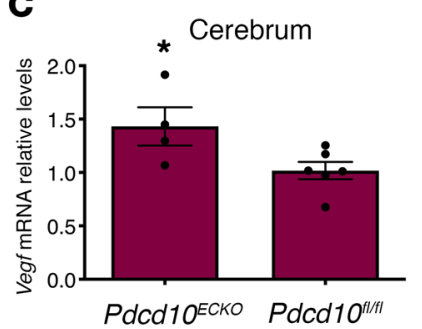

b-Gal-VEGF/GFAP
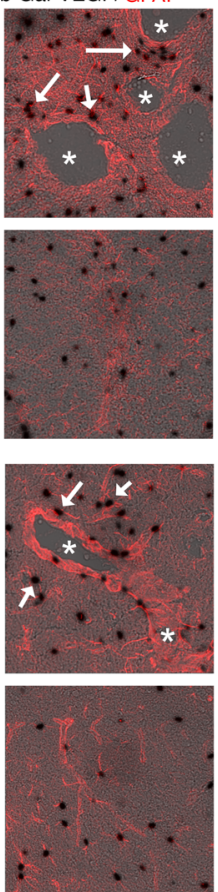
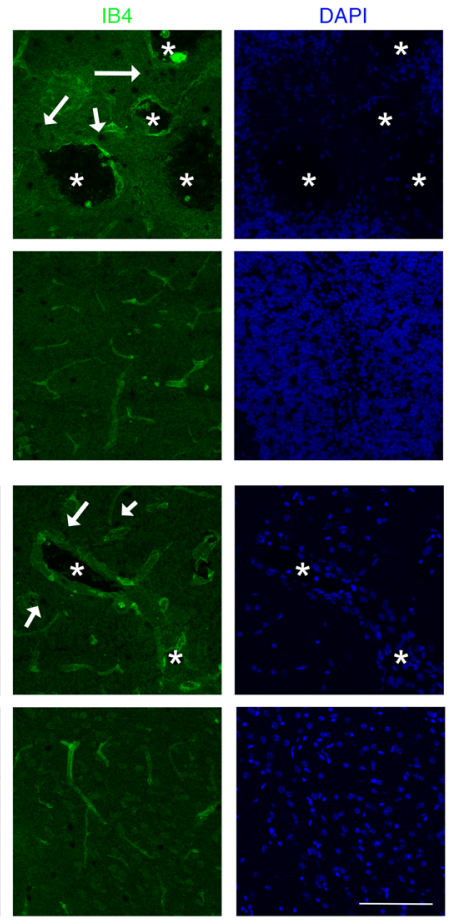

D

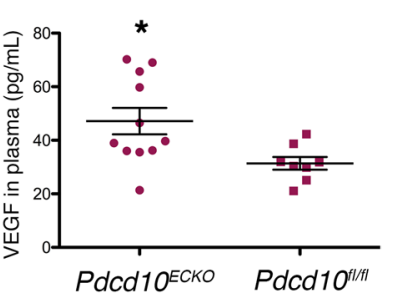

Figure 3. Astrocyte-derived VEGF increased during cerebral cavernous malformations in juvenile animal brain. (A) Confocal microscopy of brain (cerebellum and cerebrum cortex) from Pdcd10 ${ }^{\text {ECKO }}$ Vegfa ${ }^{\text {tm1.1.Nagy }}$ and Pdcd10 ${ }^{\text {fl/fl }}$ Vegfa ${ }^{\text {tm.1.1Nagy littermate controls stained }}$ for $\beta$-gal/VEGF expression detected by $X$-gal staining (black), GFAP-positive astrocytes (red), isolectin B4 (green), and DAPI for nuclear DNA (blue). Asterisks indicate vascular lumen of CCM lesions. Arrows indicate $\beta$-gal/VEGF in GFAP-positive astrocytes. (B and C) Quantification of Vegfa mRNA levels in $P d c d 10^{E C K O}$ brains when compared with littermate $P d c d 10^{f / / f l}$ controls, as assessed by RT-qPCR (SEM, $n=4$ or 6 mice in each group). (D) VEGF levels in plasma from Pdcd10 ECKO mice and littermate $P d c d 10^{f / f l}$ controls, as assessed by ELISA. $n=8$ or 11 mice in each group. Data are mean \pm SEM. ${ }^{*} P<0.05$, as determined by Student's $t$ test. Scale bar: $100 \mu \mathrm{m}$ (A).
We also observed that calbidin-positive Purkinje neurons exhibited increased $\beta$-gal/VEGF expression near CCM lesions (data not shown). Consistent with results observed using $\beta$-gal/VEGF reporter mice, high-resolution in situ hybridization (ISH) analysis confirmed an increase in VEGF expression near and around the CCM lesion colocalized with the GFAP marker of astrocytes (Figure 2B and Supplemental Figure 2). Moreover, we observed that $P d c d 1 O^{E C K O}$ hindbrains exhibited an approximately 1.8 -fold increase in Vegfa mRNA levels compared with littermate $P d c d 10^{f / f l}$ controls (Figure 2C). Administration of GCV to neonatal mice at P7 significantly reduced Vegfa mRNA levels in vascular lesions in

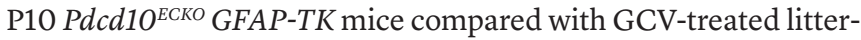
mate $P d c d 1 O^{E C K O}$ controls (Figure $2 \mathrm{C}$ ). We next tested the effect of depletion of proliferative astrocytes at the developmental stage when vascular lesions are present in $P d c d 10^{E C K O}$. Our data indicate that administration of GCV to neonatal mice between P9 to P12 significantly reduced Vegfa mRNA levels and CCM lesion burden

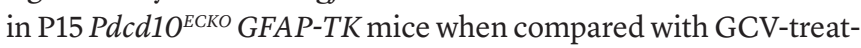
ed littermate $P d c d 1 O^{E С К O ~ c o n t r o l s ~(F i g u r e ~ 2, ~ D ~ a n d ~ E) . ~ S i n c e ~ t h e ~}$ retinal blood vessels is another vascular bed affected by the loss of $P d c d 10$ in endothelial cells, we also compared the subcellular expression of VEGF. Deletion of the endothelial Pdcd1O gene at $\mathrm{P} 3$ prominently increase $\beta$-gal/VEGF expression in $\mathrm{GFAP}^{+}$retinal astrocytes at the leading edge of blood vessel growth and imme- diately ahead of the plexus in P9 Pdcd10 ECKO Vegfa ${ }^{\text {tm1.1Nagy }}$ retinas (Supplemental Figures 2 and 3). We observed that the enhanced expression of $\beta$-gal/VEGF was associated with impaired extension of the developing superficial vascular plexus (54) in P9 Pdcd10 ЕСКО $V e g f a^{\text {tm1.1Nagy }}$ retinas when compared with $P d c d 10^{f / / f l} V e g f a^{\text {tm1.1Nagy }}$ littermate controls (Supplemental Figure 3). Moreover, defect on the extension of the vascular plexus in $\mathrm{P} 12 \mathrm{Pdcd} 1 \mathrm{O}^{\text {ECKO }}$ Vegf $\mathrm{f}^{\text {tm1.1Nagy }}$ retinas was reduced compared with $P d c d 10^{f / f l} V e g f a^{\text {tm1.1Nagy }}$ littermate controls, whereas the endothelial density and an increase in $\beta$-gal/ VEGF expression in $\mathrm{GFAP}^{+}$retinal astrocytes were elevated (Supplemental Figure 3). These data suggest that increased expression of astrocyte-derived VEGF could account for a contribution of astrocytes to CCM lesion formation.

Astrocyte-derived VEGF increases during CCM formation in juvenile mice. The previous studies showed increased VEGF expression in an acute neonatal CCM model. Recent studies have emphasized that chronic models may be more reflective of the course of the human disease $(17,55-57)$. We therefore, investigated the expression of VEGF increased in perilesional

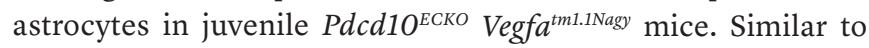
neonatal mice, we observed an increase in $\beta$-gal/VEGF expression in areas surrounding CCM lesions in P30 Pdcd10 ЕСКО Vegfa ${ }^{\text {tm1.1Nagy }}$ cerebellar tissue (Figure 3A). We found that most $\beta$-gal/VEGF ${ }^{+}$cells colocalized with the GFAP astrocyte mark- 
A

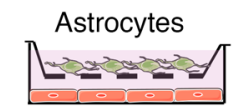

Co-cultured;

Pdcd10 ${ }^{\text {ECKO BMECs }}$

B Astrocytes in Pdcd10 ${ }^{\text {ECKO BMEC }}$
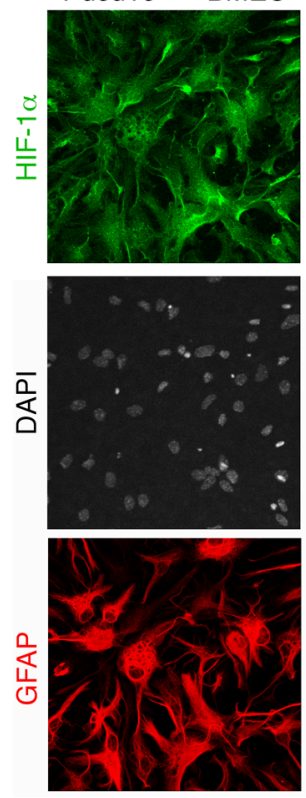

C

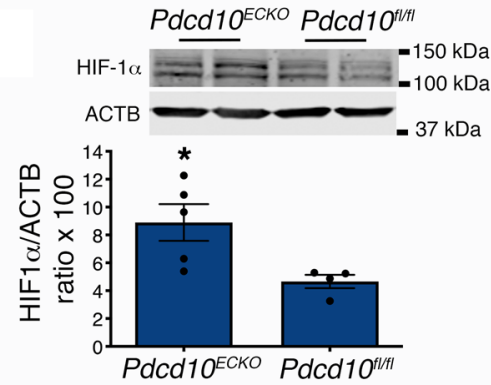

D

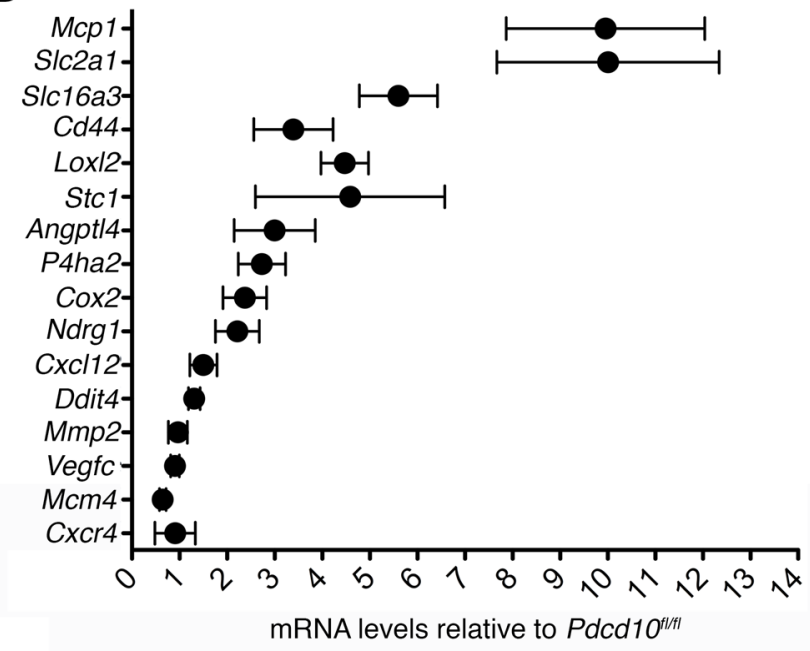

$\mathbf{E}$

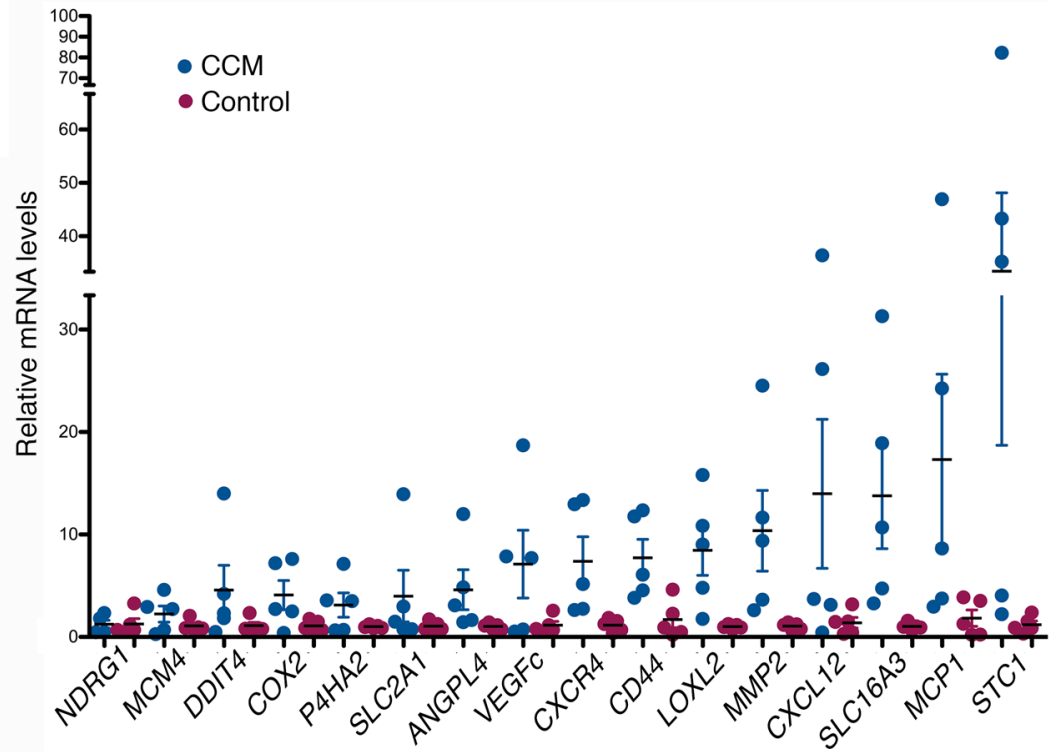

Figure 4. Increase in normoxic HIF-1 $\alpha$ stabilization in astrocytes and COX-2 during CCM. (A) Schematic diagram of astrocytes cocultured with Pdcd10ECKO and Pdcd1 ${ }^{f / f f l}$ BMECs. (B) Immunofluorescence staining for HIF-1 $\alpha$ (green), GFAP (red), and DAPI for nuclear DNA (white) of primary astrocytes cocultured with Pdcd10ECKO BMECs compared with Pdcd10fl/fI BMEC controls for 48 hours $(n=3)$. (C) Quantification of HIF-1 $\alpha$ in cerebellar tissue in P10 Pdcd10 ECKO control Pdcd1 $0^{f / f f}$ littermates, as assessed by Western blot (SEM, $n=4$ mice in each group). (D) Analysis of HIF-1 $\alpha$ target genes by RT-qPCR in cerebellar tissue from P10 Pdcd10 ${ }^{E C K O}$ and control Pdcd10 fl/fl littermates (SEM, $n=5$ or 7 mice in each group). (E) Analysis of HIF-1 $\alpha$ target genes by RT-qPCR in human CCM lesions and control human brain tissue (SEM, $n=5$ ). Data are mean \pm SEM. ${ }^{*} P<0.05$, as determined by Student's $t$ test. Scale bar: $50 \mu \mathrm{m}(\mathbf{B})$.

er in $P d c d 10^{E C K O}$ Vegfa ${ }^{\text {tm1.1Nagy }}$ mice (Figure 3A). Consistent with results observed using $\beta$-gal/VEGF reporter mice, $P d c d 10^{E C K O}$ cerebellar tissue showed a significant increase in Vegfa mRNA levels when compared with littermate $P d c d 10^{f l / f l}$ controls (Figure

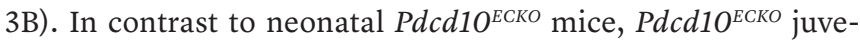
nile mice develop CCM lesions in the cerebrum $(17,55,58)$ and these were accompanied by a marked increase in $\beta$-gal/VEGF expression in $\mathrm{GFAP}^{+}$astrocytes and in $\mathrm{SOX}^{-} 9^{+}$astrocytes in $\mathrm{P} 30$

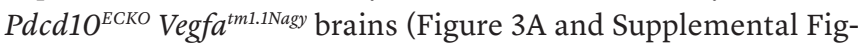
ure 3). We also observed a significant increase in Vegfa mRNA in the cerebrum of $P d c d 1 O^{E C K O}$ mice (Figure $3 \mathrm{C}$ ). Additionally, we found elevated levels of VEGF in plasma from P30 Pdcd10 ЕСКO mice when compared with $P d c d 10^{f l / f l}$ littermate controls (Figure
3D). These data support the idea that astrocyte-derived VEGF contributes to CCM lesions (32-35).

Increase in normoxic HIF-1 $\alpha$ stabilization in CCM astrocytes. Transcription factor HIF- $1 \alpha$ regulates expression of VEGF (30, 59-61). Therefore, we next investigated whether the significant increase of VEGF in astrocytes during CCMs is due to elevation of HIF- $1 \alpha$. For this study, we first prepared coculture studies to better understand the interaction between CCM endothelium and astrocytes (Figure 4A) and the level of HIF-1 $\alpha$ in purified astrocytes

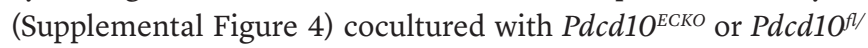
${ }^{f l}$ brain microvascular endothelial cells (BMECs) was determined. Immunocytochemistry revealed that the level of HIF-1 $\alpha$ was elevated in astrocytes cocultured with $P d c d 10^{E С К О ~ B M E C s ~ c o m p a r e d ~}$ 
A
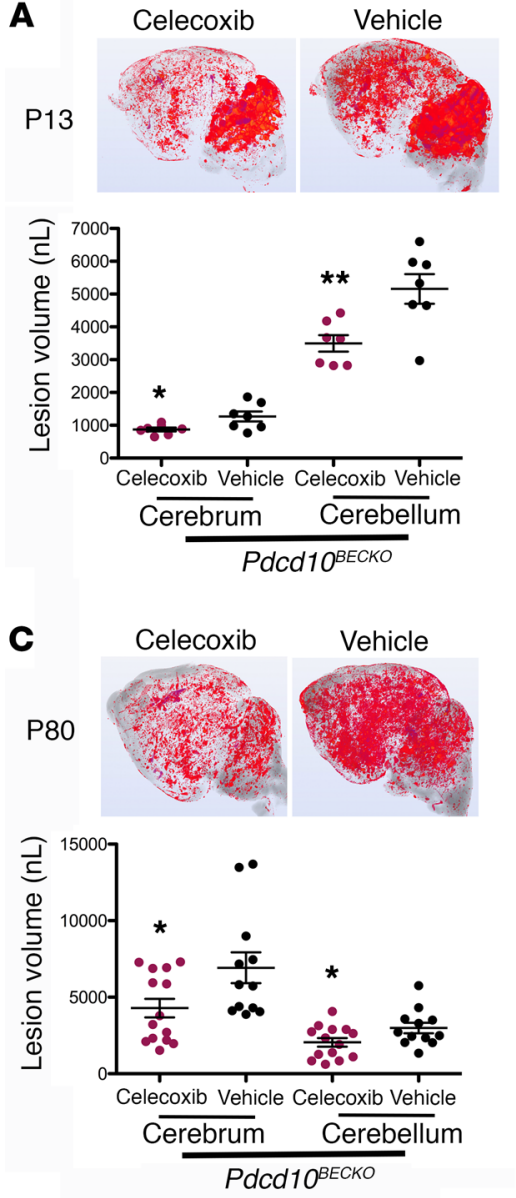

$\mathbf{E}$

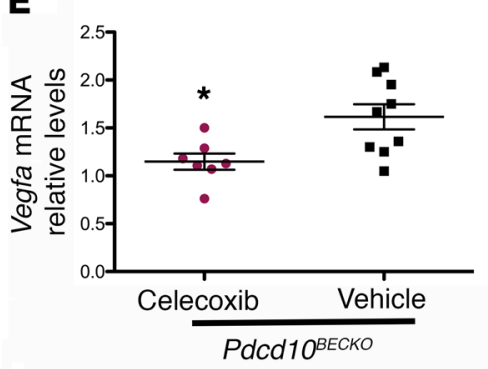

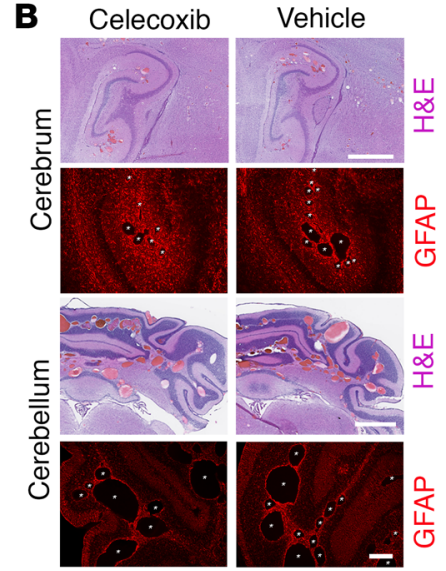

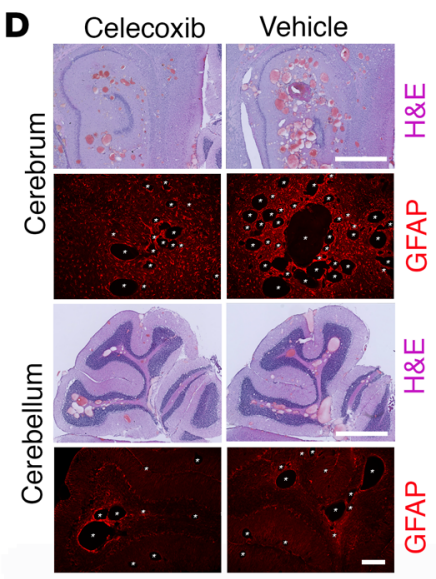

$\mathbf{F}$

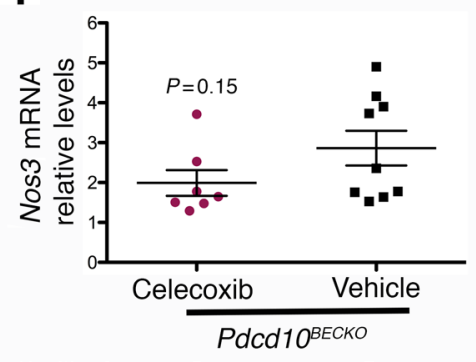

Figure 5. COX-2 inhibition prevents CCM lesions in Pdcd10 ${ }^{\text {ВЕСКо }}$ mice. (A) Prominent lesions are present in the cerebellum and cerebrum of P13 Pdcd10 ${ }^{B E C K O}$ mice. Intragastric administration of $40 \mathrm{mg} / \mathrm{kg}$ celecoxib for 4 consecutive days at P6 to P9 suppressed lesion formation. Quantification of lesion volumes by micro-CT analysis from mice at P13 treated with celecoxib or vehicle (SEM, $n=7$ mice in each group). (B) Hematoxylin and eosin (pink and purple) or GFAP (red) staining of cerebral (hippocampal area) and cerebellar sections from $P d c d 10^{B E C K O}$ mice after treatment with celecoxib or vehicle $(n=3)$. (C) Prominent lesions are present in the cerebellum and cerebrum of P80 Pdcd10 ${ }^{B E C K O}$ mice. Oral gavage administration of $40 \mathrm{mg} / \mathrm{kg}$ celecoxib for 15 consecutive days at P55 to P70 suppressed lesion formation. Quantification of lesion volumes by micro-CT analysis from mice at P80 treated with celecoxib or vehicle (SEM, $n=12$ or 14 mice in each group). (D) Hematoxylin and eosin (pink and purple) or GFAP (red) staining of cerebral (hippocampal area) and cerebellar sections from $P d c d 10^{B E C K O}$ mice after treatment with celecoxib or vehicle $(n=3)$. ( $(\mathbf{E}$ and $\mathbf{F})$ Quantification of Vegfa $(\mathbf{E})$ or Nos3 (F) mRNA levels in P80 Pdcd10 ${ }^{B E C K O}$ spinal cords after treatment with celecoxib or vehicle from experiments in (C) (SEM, $n=7$ or 9 mice in each group). Data are mean \pm SEM. ${ }^{*} P<$ $0.05,{ }^{*} P<0.01$, as determined by Student's $t$ test. Scale bars: 1 $\mathrm{mm}$ (H\&E), $200 \mu \mathrm{m}$ (GFAP) (B and D).

angiopoietin-like 4 (Angptl4) (Figure 4D). Importantly, our findings in the CCM animal model were extended to human CCM because we also detected the elevation of HIF-1 $\alpha$ regulated genes in human CCM lesions in comparison with lesion-free brain tissue from a patient with CCM or nonneurological disease control (Figure 4E). Since we observed that COX-2 is increased at mRNA (Figure 4D) and protein (data not shown) levels in Pdc-

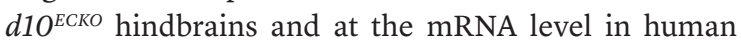
CCM lesions (Figure 4E), and it was previously showed by immunohistochemistry in human CCM tissue by Francesco Retta's group (21), we next investigated the effect of using celecoxib $(40 \mathrm{mg} / \mathrm{kg})$, a nonsteroidal antiinflammatory drug (NSAID) that primarily inhibits COX-2 activity, in 2 preclinical CCM models. In these experiments, we used brain endothelial-specific inactivation (Slco1c1-iCreERT2) of the Pdcd10 gene, where lesions are confined to the CNS and progressively form with those cocultured with control Pdcd1 $0^{f / l l}$ BMECs (Figure 4, A and B, and Supplemental Figure 4). Consistent with this obser-

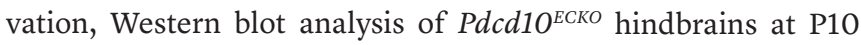
confirmed an increase in HIF-1 $\alpha$ protein levels when compared with $P d c d 10^{f / f l}$ littermate controls (Figure 4C). Furthermore, we

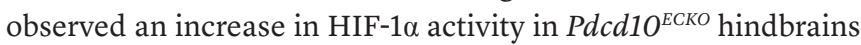
(Figure 4D). RT-qPCR analysis revealed a significant induction of HIF-1 $\alpha$ regulated genes involved in inflammation (62), including cyclooxygenase-2 (Cox2) and monocyte chemoattractant protein 1 (Mcp1) as well as genes involved in metabolic reprograming such as glucose transporter 1 (solute carrier family 2, Slc2a1, GLUT-1), lactic acid, and pyruvate transporter (solute carrier family 16, Slc16a3, also known as monocarboxylate transporter 4 MCT4) $(59,63)$. In addition, we also observed a marked increase in HIF$1 \alpha$ regulated genes involved in angiogenesis $(59,63)$, including a cell-surface glycoprotein Cd44, Lysyl oxidase-like 2 (Loxl2) and in the cerebrum and cerebellum of P13 Pdcd10 ${ }^{B E C K O}$ mice (Figure $5 \mathrm{~A})$. Blinded measurements using microCT analysis of neonatal celecoxib-treated $P d c d 10^{B E C K O}$ mice compared with vehicle-treated littermate $P d c d 1 O^{B E C K O}$ controls indicated a reduction in vascular lesion volume in both cerebellum and cerebrum (Figure 5A). Histological analysis of the hippocampal area in P13 Pdcd10 ВЕСКO mice further demonstrates the high propensity of CCM lesions to develop surrounded by $\mathrm{GFAP}^{+}$astrocytes, and inhibition of COX-2 reduced the CCM lesions' density in $P d c d 10^{\text {BECKO }}$ mice (Figure 5B and Supplemental Figure 5). Next, we investigated the

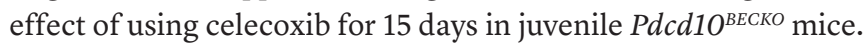
Notably, blinded measurements of microCT analysis confirmed reduction in CCM in cerebrum and cerebellum of P80 celecoxib-treated $P d c d 1 O^{\text {BECKO }}$ mice (Figure 5C). Moreover, histological analysis of the cerebellum and hippocampal area in P80 cele-

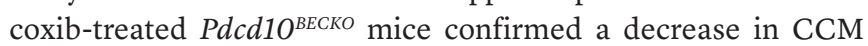


A
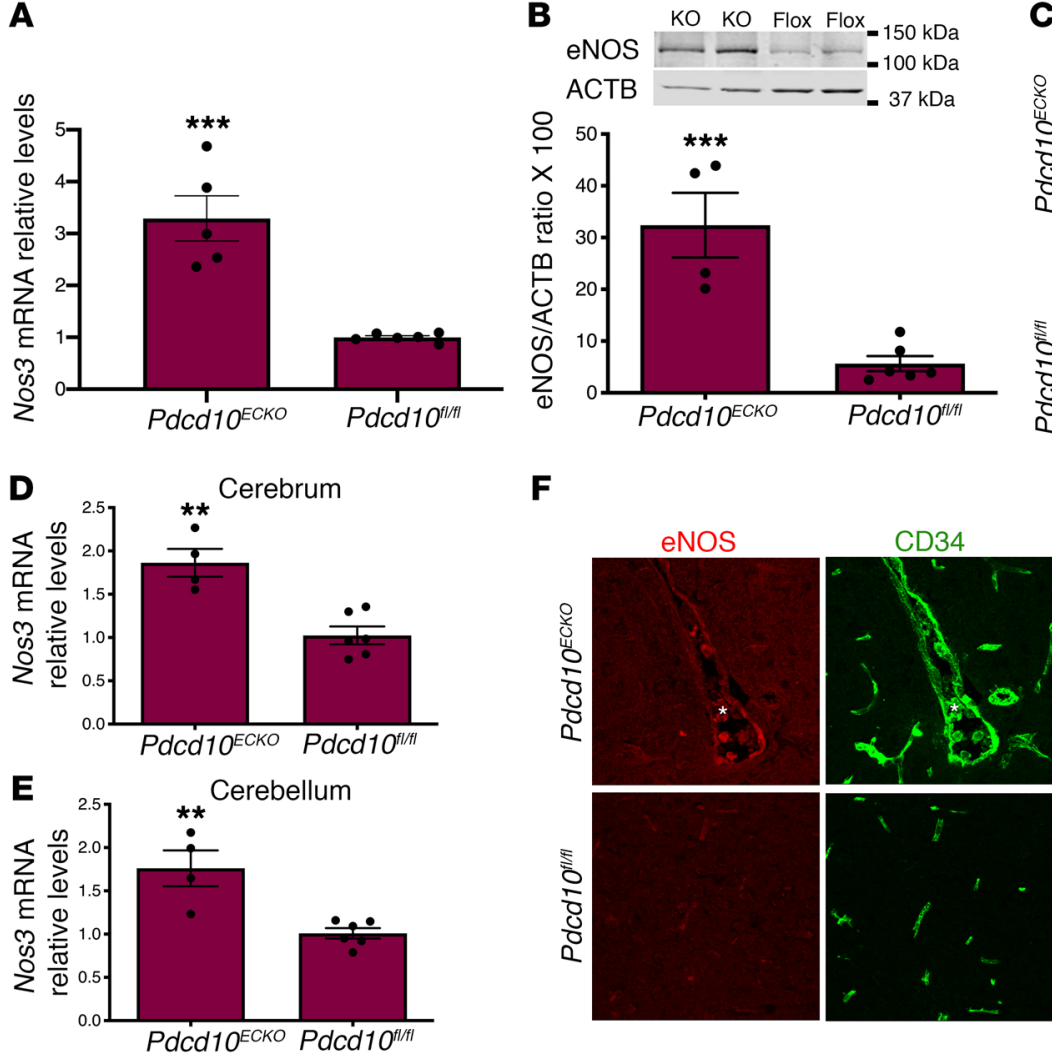
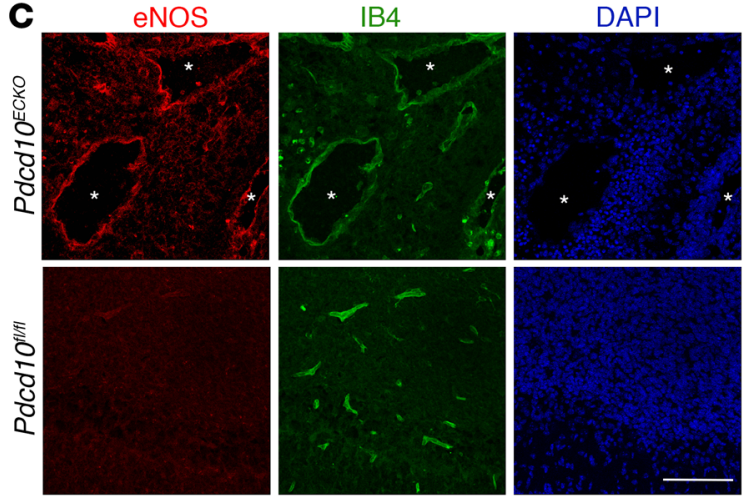
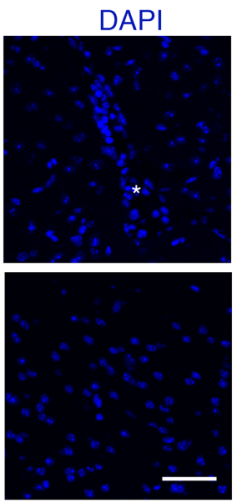

Figure 6. Loss of brain endothelial Pdcd10 increases the expression of eNOS in situ. (A) Analysis of Nos3 mRNA levels by RT-qPCR in hindbrains of P10 $P d c d 10^{E C K O}$ and littermate $P d c d 10^{f l f l}$ controls (SEM, $n=4$ or 5 mice in each group). (B) Analysis of eNOS levels in hindbrains of P10 Pdcd10 ECKO and littermate $P d c d 10^{f / f l}$ controls, as assessed by Western blot analysis (SEM, $n=4$ or 5 mice in each group). (C) Confocal microscopy of cerebellar cortex P10 stained for eNOS (red), endothelial marker isolectin B4 (green), and DAPI for nuclear DNA (blue). Asterisks indicate vascular lumen of CCM lesions ( $n=4$ mice in each group). ( $\mathbf{D}$ and $\mathbf{E})$ Analysis of Nos3 mRNA levels by RT-qPCR in brains of P30 Pdcd10 ${ }^{E C K O}$ and littermate Pdcd10fl/fl controls. (F) Immunofluorescence staining of eNOS (red), endothelial marker cd34 (green), and DAPI for nuclear DNA (blue) ( $n=4$ or 6 mice in each group). Data are mean \pm SEM. ${ }^{* *} P<0.01,{ }^{* *} P<$ 0.001 , as determined by Student's $t$ test. Scale bars: $100 \mu \mathrm{m}$ (C); $50 \mu \mathrm{m}$ (F).

lesions' density and in GFAP-immunoreactivity (Figure 5D and Supplemental Figure 5). Similar to the CCM lesions detected in brain, we observed that $\mathrm{P} 80 \mathrm{Pdcd} 1 \mathrm{O}^{\text {BECKO }}$ mice develop gross $\mathrm{CCM}$ lesions through the spinal cord (Supplemental Figure 6), and with high propensity to develop in $\mathrm{GFAP}^{+}$astrocytes areas. In addition, P80 Pdcd10 ${ }^{B E C K O}$ spinal cords exhibited an approximately 1.6-fold increase in Vegfa mRNA levels that it is prevented in P80 celecoxib-treated $P d c d 1 O^{B E C K O}$ mice (Figure $5 \mathrm{E}$ ), whereas the elevation of Nos 3 mRNA remain unchanged (Figure 5F). Collectively, these results suggest that CCM endothelium can induce HIF-1 $\alpha$-dependent hypoxia and angiogenesis programs in astrocytes and that this non-cell-autonomous mechanism contributes to disease. Moreover, the capacity of celecoxib to ameliorate 2 murine CCM models underscores the potential of therapeutic strategies to emerge from these insights.

Loss of brain endothelial Pdcd10 or Krit1 increases the expression of eNOS. Previous work has demonstrated that eNOS contributes to VEGF-induced angiogenesis and vascular permeability (44) potentially through stabilization of HIF1- $\alpha$ by NO. Genetic inactivation of Pdcd1O $\left(P d c d 1 O^{E C K O}\right)$ or Krit1 (Krit1 $\left.{ }^{E C K O}\right)$ results in increased Nos3

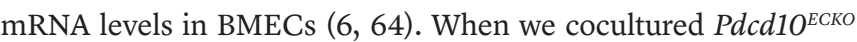
endothelial cells with astrocytes we observed a dramatic upregulation of Nos3 mRNA ( 19.2 fold increase) associated with increased
eNOS protein expression ( $~ 8.9$ fold increase) in $P d c d 10^{E C K O}$ BMECs when compared with $P d c d 10^{f / f l}$ BMEC controls as assessed by Western blot analysis (Supplemental Figure 7). Similar results were observed in Krit1 ${ }^{E C K O}$ BMECs: an approximately 7.4 fold increase Nos3 mRNA was associated with increased eNOS protein expression ( $\sim 9$ fold increase) (Supplemental Figure 7$)$. These results indicate that there is a significant increase in eNOS mRNA and protein upon genetic inactivation of $P d c d 10$ or Krit1 in BMECs.

To investigate the distribution and expression of eNOS during CCMs in vivo, we first used neonatal $P d c d 10^{E C K O}$ mice. Consistent with results observed in vitro, $P d c d 1 O^{E C K O}$ hindbrains showed an approximately 3.3 fold increase in Nos 3 mRNA levels compared with littermate $P d c d 10^{f / f l}$ controls (Figure 6A). Furthermore, Western blot analysis showed an approximately 5.8 fold increase in eNOS protein expression in $P d c d 10^{E C K O}$ hindbrains relative to $P d c d 10^{f / f l}$ hindbrains (Figure 6B). Immunohistochemistry analysis of hindbrain sections revealed that eNOS was upregulated in CCM lesions. As shown in Figure 6C, we observed that eNOS staining was increased in $P d c d 1 O^{E C K O}$ dilated vasculature as showed by colocalization of antibodies specific against eNOS and isolectin B4-FITC staining, which specifically labels the brain vasculature (Figure 6C). Moreover, analysis of the distribution and expression of eNOS in a juvenile CCM animal model indicated that Nos3 
A

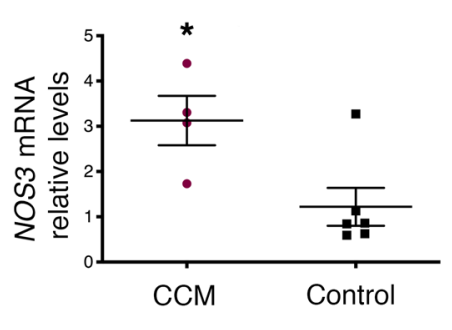

B

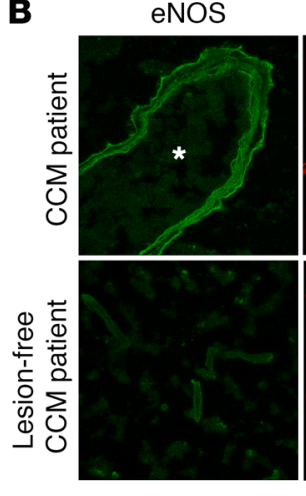

Col IV

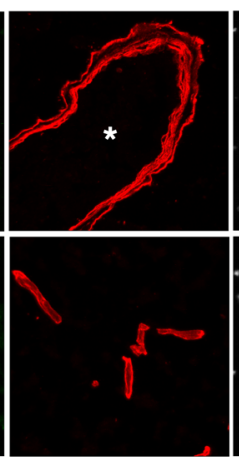

DAPI

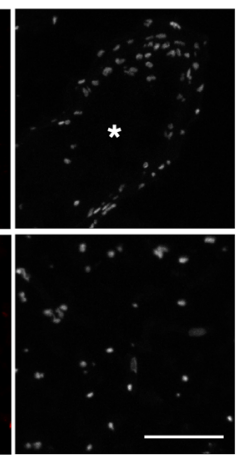

C

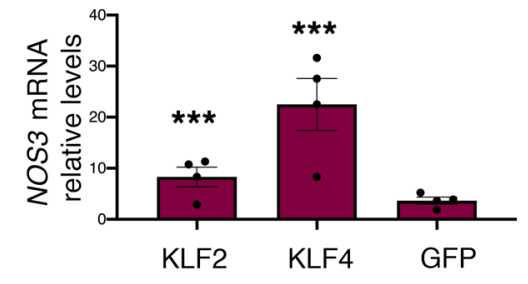

$\mathbf{F}$

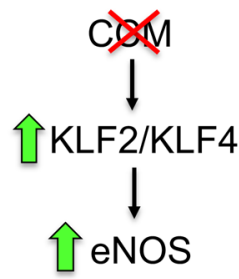

D

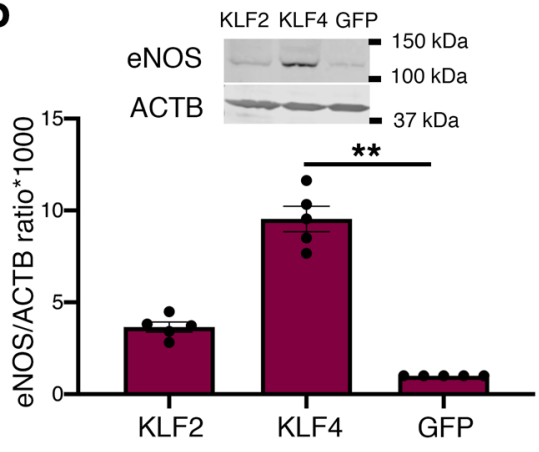

$\mathbf{E}$

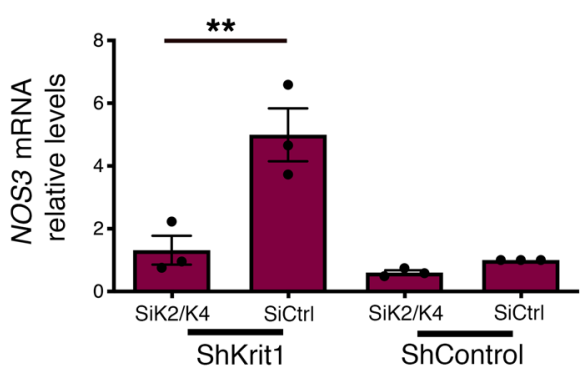

Figure 7. KLF2 and KLF4 regulate increased eNOS expression during CCM. (A) Expression levels of NOS3 mRNA as assessed by RT-qPCR from human CCM lesions and compared with nonneurological disease controls (SEM, $n=4$ or 6 in each group). (B) Immunofluorescence staining of eNOS (green) and collagen IV (Col IV; red) of human CCM lesion matched to CCM lesion-free brain tissue $(n=3)$. Asterisks denotate vascular lumen of CCM lesion. Nuclei were counterstained with DAPI (white). (C) Human umbilical vein endothelial cells (HUVECs) were transduced with lentivirus encoding KLF2 or KLF4, as previously reported (6), and analysis of NOS3 mRNA levels by RT-qPCR was determined in cells overexpressing KLF2 or KLF4 and compared with lentivirus encoding GFP as a control ( $n=3$ or 4). (D) Analysis of eNOS protein levels in HUVECs transduced with lentivirus encoding KLF2 or KLF4, as determined by Western blot analysis (40); lentivirus encoding GFP was used as a control (SEM, $n=3$ ). (E) Analysis of NOS3 mRNA levels by RT-qPCR in hCMEC/D3 cells transduced with lentivirus encoding shKRIT1 or scrambled control, followed by transfection with KLF2- and KLF4-specific small interfering RNAs (siRNA; siK2/K4) or small interfering RNA control (siCtrl) (SEM, $n=4$ ). (F) Schematic model. KLF2- and KLF4-mediated elevation of eNOS in CCM endothelium by a cell-autonomous mechanism. Data are mean \pm SEM. ${ }^{* *} P<0.01,{ }^{* *} P<0.001$, as determined by Student's $t$ test and 1-way ANOVA, followed by the Tukey post hoc test. Scale bar: $100 \mu \mathrm{m}$ (B).

mRNA levels were elevated in brain tissue in $P d c d 10^{E С К О ~ m i c e ~(F i g-~}$ ure 6, D and E). We also observed robust and focal upregulation of eNOS protein in the lesions of $P d c d 10^{E C K O}$ mice (Figure $6 \mathrm{~F}$ ). Together, these results demonstrate that eNOS mRNA and protein expression is increased in CCM lesions.

Increased eNOS expression in human CCMs is regulated by KLF2 and KLF4. In human CCM brain tissue, there was an approximately 3 fold increase in NOS3 mRNA levels in comparison to lesion-free brain tissue (Figure 7A). We also observed that eNOS staining was increased in human CCM endothelium in comparison with lesionfree brain tissue (Figure 7B). Transcription factors KLF2 and KLF4 are regulators of eNOS $(65,66)$. Therefore, we confirmed that the changes in brain endothelial eNOS, at the protein and mRNA levels, were associated with an increase in transcription factors KLF2 and KLF4 (Figure 7, C and D). Moreover, we observed that reducing the expression of KLF2 and KLF4 (40) prevented increased eNOS expression in KRIT1-depleted human brain endothelial cells (Figure 7E). These results suggest that the increase in KLF2 and KLF4 increased brain endothelial eNOS in CCMs (Figure 7F).

Loss of brain endothelial Pdcd1O increases NO production via eNOS. Endothelial cells metabolize L-arginine via eNOS to produce NO, therefore we next investigated if eNOS upregulation increases NO production in $P d c d 10^{E C K O}$ BMECs. Using a colorimetric assay to quantitatively measure total $\mathrm{NO}_{2}$ and $\mathrm{NO}_{3}$ (nitrites and nitrates) in the culture media, we observed that $P d c d 1 O^{E С K O}$ BMECs possessed an approximately 1.7 fold increase in NO release when compared with $P d c d 10^{f / f l} \mathrm{BMEC}$ controls (NO basal levels: $\sim 3 \mu \mathrm{M}$ ) (Figure $8 \mathrm{~A}$ ). The increase in NO release is ascribable in part to the upregulation of eNOS, because genetic inactivation in one copy of the Nos3 gene $\left(\mathrm{Nos}^{+/-}\right)$significantly reduced NO production in $P d c d 10^{E C K O}$ BMECs (Figure 8B). Consistent with these results, low levels of Nos3 mRNA (data not shown) and eNOS protein in $\mathrm{Pdcd} 1 \mathrm{O}^{\text {EСКO }} \mathrm{Nos}^{+/-}$BMECs were observed (Figure $8 \mathrm{C}$ ). Moreover, addition of a NO synthase (NOS) inhibitor, L-NAME, reduced NO production (Figure $8 \mathrm{~B}$ ) without changing eNOS mRNA and protein levels (data not shown). Taken together, these studies show that the loss of Pdcd1O in BMECs leads to an increase in eNOS expression and subsequent endothelial NO release.

Brain endothelial NO induces astrocyte-derived VEGF following loss of Pdcd10. We showed evidence of increased levels in VEGF expression in $\mathrm{GFAP}^{+}$brain and retinal astrocytes in $P d c d 1 O^{E C K O}$ mice (Figure 2, A, C, D; Figure 3, A and B; and Supplemental Figures 2 and 3). However, our in vivo experiments did not unequivocally exclude expression of VEGF in other CNS-resident cells during 
A

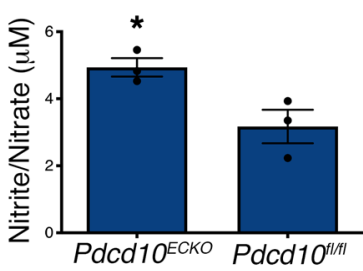

D Co-cultured; Pdcd10 $0^{E C K O}$ or Pdcd10 $0^{\prime \prime H I}$ BMECs

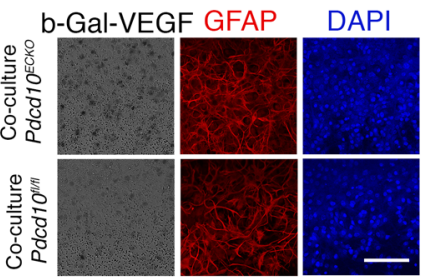

G
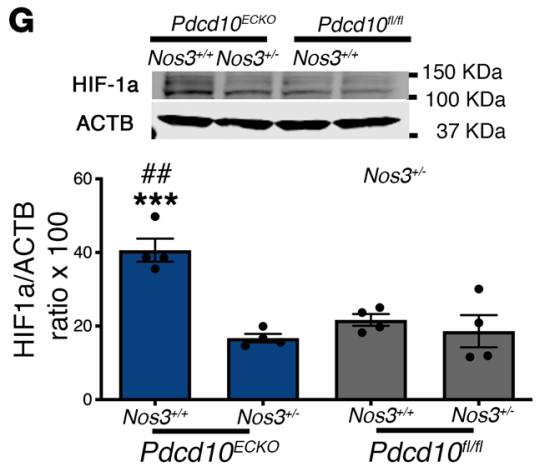

I

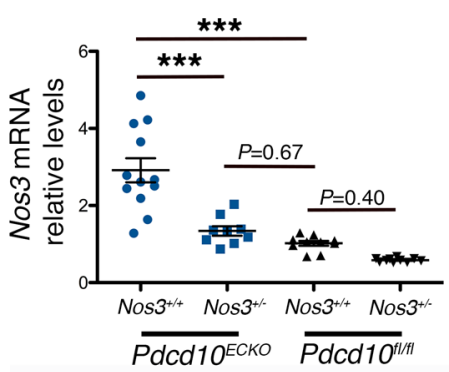

B
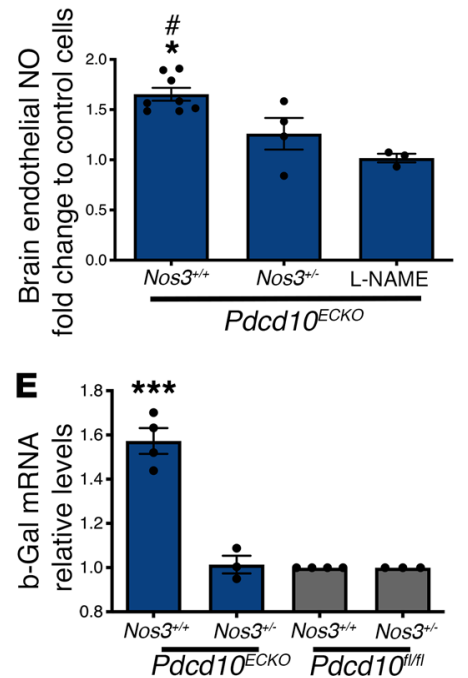

C

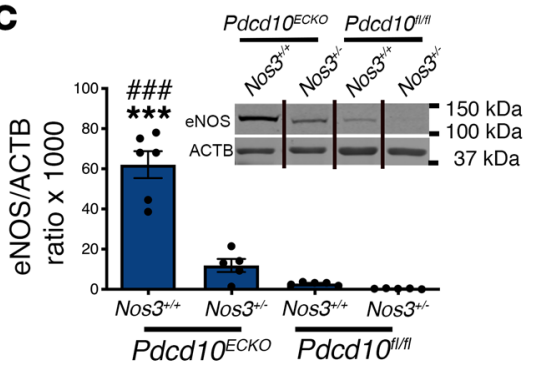

$\mathbf{F}$

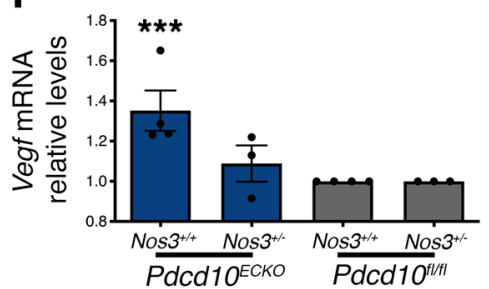

H

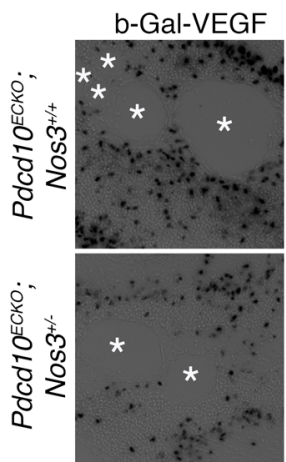

J

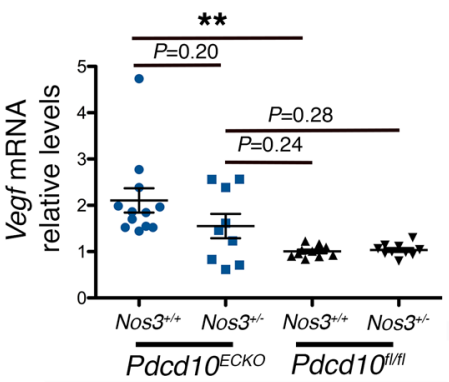

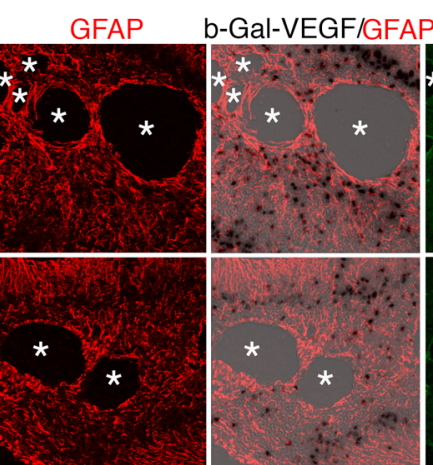

Pdcd10 $0^{\text {BECKO }}$

$\mathbf{K}$

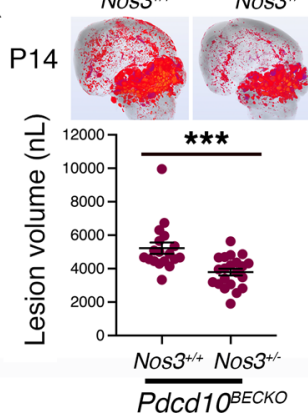

$\mathbf{L}$

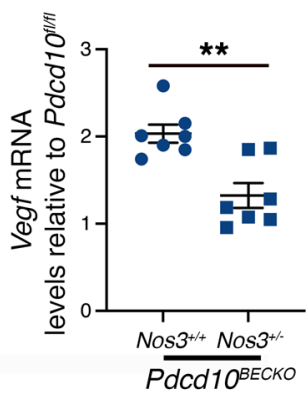

Figure 8. Loss of brain endothelial Pdcd10 increases NO production and induces astrocyte-derived VEGF. (A) Total NO production from the media of $P d c d 10^{E C K O}$ and $P d c d 10^{f l / f l}$ BMECs cultured for 36 hours (SEM, $n=7$ ). (B) NO release in Pdcd10 ECKO and Pdcd10ECKO Nos $3^{+/-}$BMECs or following incubation

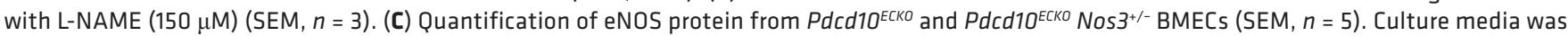
supplemented with $500 \mathrm{uM} \mathrm{L-arginine} \mathrm{and} \mathrm{was} \mathrm{deficient} \mathrm{in} \mathrm{serum.} \mathrm{Lanes} \mathrm{in} \mathrm{this} \mathrm{panel} \mathrm{were} \mathrm{run} \mathrm{on} \mathrm{the} \mathrm{same} \mathrm{gel} \mathrm{but} \mathrm{were} \mathrm{noncontiguous.} \mathrm{(D)} \beta$-gal/ VEGF expression (black) and staining for GFAP (red) of primary cultured astrocytes cocultured with Pdcd10 ${ }^{E C K O}$ BMECs compared with Pdcd10flff BMEC controls for 48 hours $(n=2)$. (E) RT-qPCR analysis of $\beta$-gal and $(\mathbf{F})$ Vegfa mRNA in primary cultured astrocytes cocultured with Pdcd10 ECKO BMEC compared with Pdcd10 $0^{f l / f l}$ BMEC controls (SEM, $\left.n=4\right)$. (G) Quantification of HIF-1 $\alpha$ protein from primary astrocytes cocultured with Pdcd10 ${ }^{E C K O}$ BMEC and

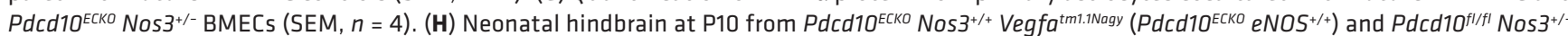
Vegfat ${ }^{\text {tm1.1Nagy }}$ (Pdcd10 ECKO eNOS+/-) littermate controls stained for GFAP-positive astrocytes (red), $\beta$-gal/VEGF (black), isolectin B4 (green). Asterisks indicate vascular lumen of CCM lesions $(n=3)$. (I and $\mathbf{J})$ Quantification of Nos3 $(\mathbf{I})$ and Vegf $(\mathrm{J})$ mRNA levels in P10 Pdcd10ECKO Nos $3^{+/-}$and Pdcd10ECKO and Pdcd1 $0^{f / / f l} \mathrm{Nos}^{+/-}$hindbrains when compared with littermate Pdcd10 $0^{f / f l}$ controls, as assessed by RT-qPCR (SEM, $n=9$ or 12 mice in each group).

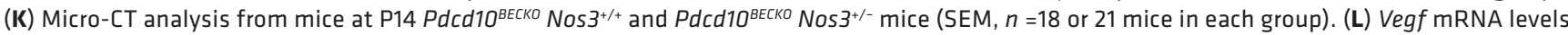

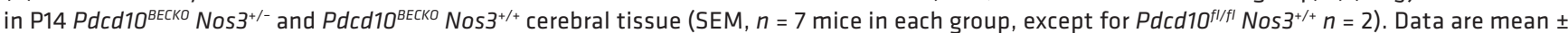

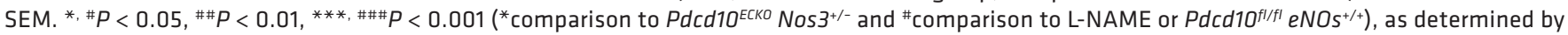
Student's $t$ test and 1-way ANOVA, followed by the Tukey post hoc test. Scale bars: $100 \mu \mathrm{m}$ ( $\mathbf{D}$ and $\mathbf{H}$ ). 


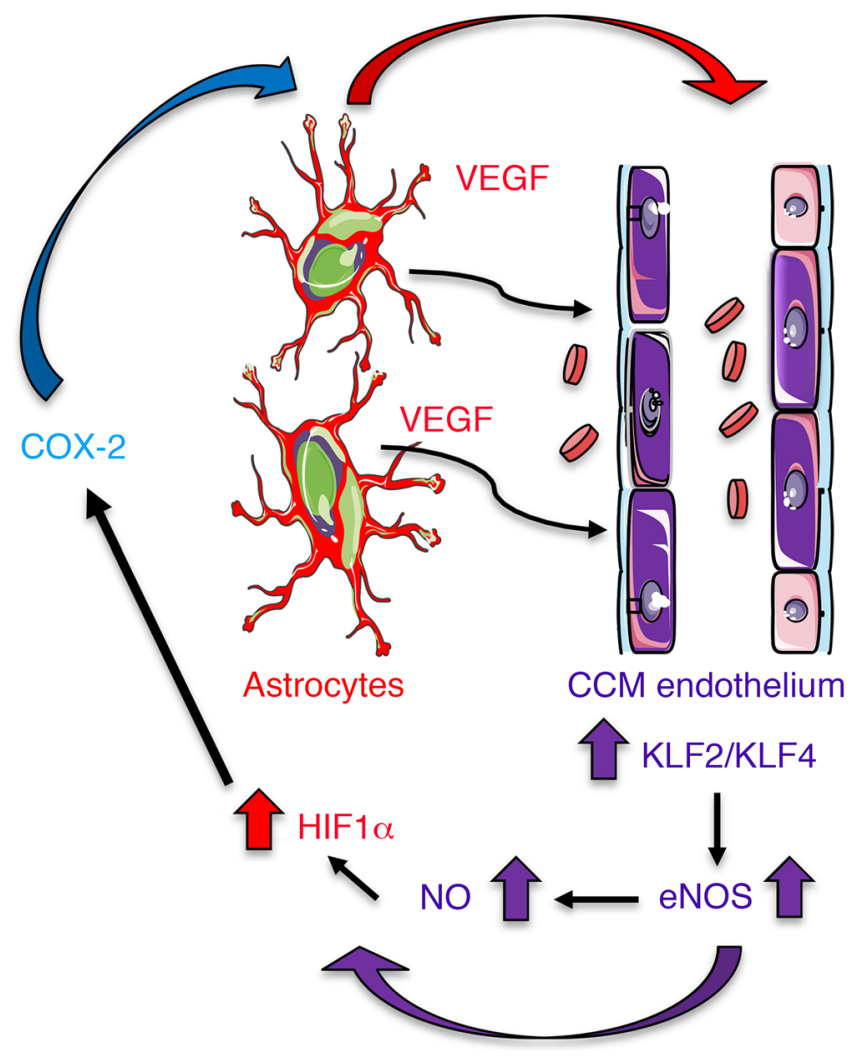

CCMs, including neurons, microglia, and pericytes. Thus, we complemented those studies by performing coculture experiments between CCM endothelium and astrocytes. First, NO donors, such as DETA-NONOate, promote brain angiogenesis by upregulation of VEGF signaling (46). To investigate whether NO stimulates the expression of VEGF in astrocytes during CCMs, we prepared primary mouse astrocyte cultures from Vegfa $a^{\text {tml.1Nagy }}$ mice (Figure 8D). We observed that the purity of cultured astrocytes was high, as determined by cells that are double positive for specific astrocyte markers GFAP and integrin B5 (Supplemental Figure 4). We also observed that our primary astrocyte cultures respond to exogenous NO (0.5 mM DETA-NONOate [NO donor]) and that elevated levels of $\mathrm{NO}$ can increase astrocyte-derived VEGF (as assessed by increase in $\beta$-gal/VEGF expression) (Supplemental Figure 8). We next investi-

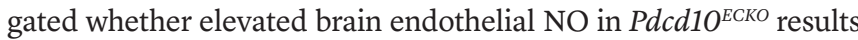
in increased astrocyte-derived VEGF levels. Therefore, we cocultured purified astrocytes in the presence of $P d c d 10^{E C K O}$ or $P d c d 10^{f / f l}$ BMECs in serum-free conditions supplemented with L-arginine, and measured changes in $\beta$-gal/VEGF expression in astrocytes. We observed that $P d c d 10^{E С К O ~ B M E C s ~ s i g n i f i c a n t l y ~ i n c r e a s e ~} \beta$-gal/ VEGF expression in astrocytes in a coculture system (Figure 8, D and E). These results were consistent with increased levels of $\beta$-gal and Vegfa mRNA levels in astrocytes cocultured with $P d c d 1 O^{E C K O}$ BMECs compared with those cocultured with $P d c d 10^{f / f l}$ BMEC controls (Figure 8, E and F). Furthermore, genetic inactivation of 1 copy of the Nos3 gene in Pdcd1O ECKO BMECs was sufficient to prevent stability of HIF-1 $\alpha$ and subsequent Vegfa/ $\beta$-gal upregulation in astrocytes, indicating that the increase in astrocyte-derived Vegfa/ $\beta$-gal mRNA levels was specific to the upregulation of eNOS (Figure 8, E and F) and dependent on HIF-1 $\alpha$ stability (Figure $8 G$ ).
Figure 9. Astrocytes integrate a circuit of neurovascular dysfunction during CCM disease. Model by which astrocytes make a substantial contribution to CCM pathogenesis. An increase in astrocyte VEGF synthesis is driven by endothelial NO generated due to KLF2- and KLF4-dependent elevation of eNOS in CCM endothelium. Production of NO in CCM endothelium stabilizes HIF-1 $\alpha$ in astrocytes, resulting in increased VEGF production and expression of a hypoxic program under normoxic conditions. Pharmacological inhibition of HIF1-driven COX-2 can ameliorate murine models of CCM disease.

We next investigated whether the increase in astrocyte-derived VEGF during CCMs was associated with elevation of eNOS in mice. Vegfat $a^{\text {tm1.1Nagy }}$ mice were intercrossed with CCM animal models and with animals deficient in 1 copy of the Nos 3 gene $\left(\mathrm{eNOS}^{+/-}\right)$. We observed by histology that CCM lesions were slightly reduced and with a partial decrease in $\beta$-gal/VEGF expression in P10 Pdcd10 ECKO Vegfa ${ }^{\text {tm1.1Nagy }} \mathrm{Nos}^{+/-}$hindbrains when compared with $\mathrm{Pdcd10} \mathrm{O}^{\text {ECKO }}$ Vegfa ${ }^{\text {tm1.1Nagy }} \mathrm{Nos}^{+/+}$littermate controls (Figure $8 \mathrm{H})$. We confirm that the increased levels of Nos3 mRNA were significantly prevented in $\mathrm{Pdcd} 1 \mathrm{O}^{\text {ECKO }} \mathrm{Nos}^{+/-}$hindbrains when compared with Pdcd $1 \mathrm{O}^{E C K O} \mathrm{Nos}^{+/+}$littermate (Figure 8I). However, we also noted that levels of Nos3 mRNA tend to be higher in

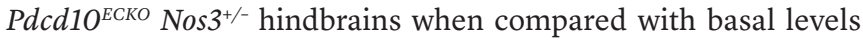
in $\mathrm{Pdcd} 1 \mathrm{O}^{\mathrm{fl} / \mathrm{fl}} \mathrm{Nos}^{+/+}$littermate controls (Figure 8I). Consistent with results observed by histological analysis, $\mathrm{Pdcd} 1 \mathrm{O}^{\text {ECKO }} \mathrm{Nos}^{+/-}$ hindbrains showed a trend to reduced levels of Vegf mRNA and lesion genesis when compared with $\mathrm{Pdcd} 1 \mathrm{O}^{\mathrm{ECKO}} \mathrm{Nos}^{+/+}$littermate (Figure 8J and Supplemental Figure 9). Notably, we observed a trend in the increase of Vegf mRNA levels in the cerebellar tissue

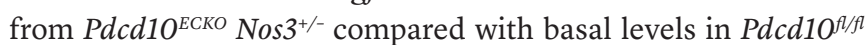
$\mathrm{Nos}^{+/+}$or $\mathrm{Pdcd} 1 \mathrm{O}^{f l / f l} \mathrm{Nos}^{+/-}$littermate controls (Figure 8J). We next assessed CCM lesions using brain endothelial-specific inactivation (BECKO, Slco1c1-iCreERT2 line) of the Pdcd1O gene, where lesions initially form in the cerebellum (P6-P7) but pro-

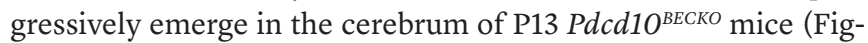
ure 5A). Blinded measurements using microCT analysis from P14 $P d c d 10^{\text {BECKO }} \mathrm{Nos}^{+/-}$whole brains and compared with brains in P14 Pdcd10 ${ }^{\text {ВЕСКО }} \mathrm{Nos}^{33^{+/+}}$mice indicate a significant reduction in brain vascular lesions volume (Figure $8 \mathrm{~K}$ and Supplemental Figure 9). Moreover, we also observed that genetic inactivation of 1 copy of the Nos3 gene in $\mathrm{Pdcd} 1 \mathrm{O}^{\text {BECKO }}$ mice ( $\mathrm{Pdcd} 1 \mathrm{O}^{\mathrm{BECKO}} \mathrm{Nos}^{+/-}$) was sufficient to prevent Vegfa mRNA upregulation in cerebrum (front of brain) that correlates with a significant decrease in cerebral lesions (Figure 8, K and L). These results indicate that eNOS/ NO signaling contributes to the elevation in VEGF expression and lesion genesis in $P d c d 10^{E C К O ~ h i n d b r a i n s ~(F i g u r e ~ 8, ~ I ~ a n d ~} \mathrm{J}$ ) and $P d c d 10^{B E C K O}$ forebrains (Figure 8, K and L).

Altogether our data provide key insights into understanding a circuit of neurovascular dysfunction during CCM disease triggered by astrocyte-endothelial crosstalk (Figure 9). These data provide strong evidence that the sustained NO production as a consequence of the elevation of eNOS in CCM endothelium, through elevation of KLF2/KLF4, contributes to the normoxic stability of HIF-1 $\alpha$ and subsequent elevation in astrocyte-derived VEGF. Therefore, astrocytes play a critical role in CCM pathogenesis by expressing hypoxia-related genes. This work establishes the principle that reciprocal communication between 
CCM endothelium and astrocytes drives lesion development. Our findings further support that the hypoxic program components, such as COX-2, represent potential therapeutic targets for CCM disease (Figure 9).

\section{Discussion}

The propensity of CCM lesions to form in the CNS parenchyma has never been mechanistically clarified. Our study shows that astrocytes respond to CCM endothelium to initiate a non-cell-autonomous enhancement of CCM formation mediated by astrocyte hypoxia and angiogenesis programs. Our experiments show that elevation of eNOS in CCM endothelium contributes to increase in astroglia-derived VEGF and HIF-1 $\alpha$ protein stabilization in astrocytes during CCM disease. The increase in eNOS expression was ascribable to an elevation of the KLF2 and KLF4 transcription factors in CCM. eNOS production in the CCM endothelium results in a sustained elevation of NO that leads to normoxic HIF-1 $\alpha$ protein stabilization and increase in HIF activity in CCM tissue. We therefore propose that astrocytes contribute to CCM disease by a non-cell-autonomous mechanism mediated by CCM endothelium-driven hypoxia and angiogenic programs.

Our in vivo observations in the neonatal CCM animal models, using endothelial-specific inactivation of $P d c d 10$ or Krit1, revealed that lesion development is spatially restricted to zones of fibrous astrocytes, which are prevalent in the white matter of the CNS (49), and that the selective ablation of proliferative astrocytes (51) markedly reduced CCM lesion and an increase in VEGF expression, prompting the suggestion that a subset of proliferative astrocytes supports CCM lesions in the developing hindbrain. Moreover, our observations using brain endothelial-specific inactivation of $P d c d 10$ (67), further revealed the high propensity of CCM lesions to develop surrounded by $\mathrm{GFAP}^{+}$ astrocytes throughout the CNS, including the cerebellum, cerebrum, and spinal cord. Earlier reports have shown that loss of $P d c d 10$ in neuroglia cells leads to formation of CCM lesions through non-cell-autonomous mechanisms, implicating a role of neural cells in the CCM pathogenesis (20). Consistent with these findings, astrogliosis has been shown to intermingle with granulation tissue in human CCM (68). However, while some subsets of reactive and proliferative astrocytes can be detrimental and contribute to CNS pathologies while other subsets of reactive astrocytes can be beneficial by supporting CNS recovery $(27,51,69$, 70). The mechanisms that contribute to functional heterogeneity in reactive astrocytes are not completely understood. Recent studies have shown that microglia control astrocyte pathogenic activities during neuroinflammation by releasing of VEGF- $\beta$ and TGF- $\alpha$ (71), and during neurodegeneration by secreting of IL- $1 \alpha$, TNF and C1q (27). Our findings revealed that the CCM endothelium controls astrocyte responses through increasing the levels of eNOS and subsequent release of brain endothelial NO. Vascular eNOS has important roles in regulating endothelial homeostasis by producing tonic NO levels, but upregulation of the eNOS gene or activated form has been previously implicated in vascular permeability, angiogenesis, and neuroinflammation (29, 45, 46, 72). Here we show that eNOS is increased in human CCM endothelium and the upregulation in endothelial KLF2 and KLF4 can account for the augmented eNOS expression at the transcription- al level (66). However, these studies do not exclude the possibility that the elevation of endothelial VEGF signaling during CCM (6) may also contribute to a sustained eNOS activation $(44,73)$ by posttranslational modification via PI3K/AKT, $\mathrm{Ca}^{2+} /$ calmodulin signaling (74) during long-term adaptations, and this notion needs to be further tested.

CCMs are hypersensitive to angiogenesis due to increase in VEGF signaling, the loss of an antiangiogenic checkpoint protein, TSP1 $(6,41)$, augmented secretion of angiopoietin-2 (11), and deregulation of Notch signaling (75). The increase in VEGF signaling may be associated with elevated levels of angiogenic factor VEGF in CCM lesions of individuals with the hereditary and sporadic form of the disease (32-35). We found that CCM endothelium-induced elevation of astrocyte-derived VEGF in neonatal, juvenile brains and adult spinal cords, and elevation of VEGF levels are dependent on eNOS signaling. Notably, an increase in astrocyte-derived VEGF can contribute to the development and progression of CNS disease (29-31) by disassembly of interendothelial junctions $(6,76,77)$. Importantly, early studies show that eNOS contributes to VEGF-induced angiogenesis through the intercellular messenger NO (44-46, 78). Furthermore, it has also been shown that NO-mediated astrocyte HIF-1 $\alpha$ stabilization modulates transcriptional and metabolic activities $(78,79)$. In agreement with this, we observed elevated levels of eNOS protein and NO bioavailability in $P d c d 10^{E C K O}$ BMECs that increased levels of HIF- $1 \alpha$ and VEGF in cocultured astrocytes. Moreover, loss of 1 copy of the gene Nos 3 in Pdcd10 ECKO BMECs is sufficient to attenuate astrocyte HIF- $1 \alpha$ stabilization and VEGF expression in the coculture system. In addition, using animal models of CCM disease we also observed that loss of 1 copy of Nos3 gene significantly reduces vascular lesions volume that was associated with decrease in levels of Nos3 and Vegf mRNA. Paradoxically, mice with loss of 2 copies of Nos3 gene ( $P d c d 1 O^{\text {ECKO }}$ $\mathrm{Nos}^{-/-}$mice) were not protected from CCM and instead showed early lethality (data not shown). The latter findings are consistent with previous reports in which a tonic amount of endothelial NO is responsible for the homeostasis between endothelium and surrounding parenchyma (72). Indeed, endothelial dysfunction in $\mathrm{Nos}^{-1-}$ mice is well-documented in disease models (80). On the $\mathrm{Nos}^{-/-}$background, mice show an increase in leukocyte-endothelial interaction, platelet aggregation, thrombosis, gliosis, and vascular permeability, among other changes in these disease models (81-84). In contrast, the endothelial dysfunction in those models is not observed in the $\mathrm{Nos}^{+/-}$mice. Thus, we ascribe the lack of protection in $\mathrm{Nos}^{-/-}$mice to the resulting endothelial dysfunction on the $P d c d 1 O^{E C K O}$ background.

Importantly, although we found that astrocytes respond to CCM endothelium, other cells of the neurovascular unit (e.g., neurons, pericytes, and microglia) may also contribute to CCM disease in a non-cell-autonomous manner through normoxic stabilization of HIF- $1 \alpha$ protein. It is tempting to speculate that CCM endothelium may harmonize cell activation in the neurovascular unit by elevation of $\mathrm{NO}$ during $\mathrm{CCM}$ disease. Because NO can reach cells millimeters away from where it is produced, this in turn can stabilize HIF-1 $\alpha$ protein $(85,86)$ from degradation by several mechanisms directly by HIF-1 $\alpha$ nitrosylation or also indirectly by increasing production of reactive oxidative 
species or by binding to the iron cofactor required for prolyl hydroxylases activity (87). HIF-1 $\alpha$ can translocate to the nucleus where it dimerizes with HIF-1 $\beta$ and binds to the hypoxia-responsive element in the promoter region of several target genes (63). Our data indicate that in mouse and human CCM tissue, the HIF-1 $\alpha$ program associated with the glycolytic pathway is activated by an increase in GLUT-1, which guarantees rapid energy production, and there is an increase in MCT4 that promotes efficient removal of lactic acid, an end product of glycolysis metabolism (63). Elevated HIF activity in CCM tissue was also associated with increases in several proangiogenic and inflammatory factors produced by glial cells, including ANGPTL4, which can induce vascular dysfunction in CNS pathologies by triggering angiogenesis $(88,89)$, and COX-2, an enzyme that catalyzes the biosynthesis of prostanoids during inflammation (62). We focused on studying COX-2 inhibition during CCM because it has been shown that COX-2 inhibition can suppress angiogenesis in vitro and in vivo $(90,91)$. Moreover, selective COX-2 inhibitors are safe and well-tolerated drugs that can be repurposed for therapy for CCM disease. Our results show that pharmacological inhibition of COX-2 significantly reduced vascular lesion formation in 2 models of CCM disease. Importantly, we noticed that the inhibition of COX-2 in the chronic CCM animal model results not only in a decrease in the density of CCM lesions but also in GFAP-immunoreactivity, suggesting that amelioration of lesion genesis counterparts astrocyte activity in the lesions. In line with these results, a recent retrospective cohort study reported that the use of NSAIDs was correlated with lower risk of hemorrhage among patients affected by CCMs (92). However, this uncontrolled association cannot be interpreted as proof of therapeutic benefit, as patients with recent CCM hemorrhages were inherently less likely to be taking NSAIDs. Cox-2 inhibitors must be investigated for safety and effectiveness in prospective controlled trials, and specific dose-effect and duration of treatment must be carefully defined. A platform for trial readiness, exploring proof-of-concept effect on lesional bleeding in human subjects, is currently being developed, and can efficiently be applied to repurposed drugs, such as NSAIDs (93). The precise role of NSAIDs in reducing CCM risk of hemorrhage version lesion formation remains incompletely understood. The COX-2/prostaglandin pathway has been implicated in vascular sprouting, migration, and induction of growth factors and proteolytic enzymes that alter angiogenesis $(90,91,94,95)$, suggesting that COX-2 could be an important therapeutic target for pathological angiogenesis $(21,90)$. Moreover, inhibition of COX-2-augmented VEGF pathway blockade during refractory tumor angiogenesis (96) suggests that combinatorial COX-2/ VEGF pathway inhibition could be used as a potential approach to ameliorate or prevent CCMs. In addition, Sulindac metabolites, an NSAID, has been shown to inhibit b-catenin-stimulated transcription of endothelial-mesenchymal transition markers and development of CCMs (97). However, additional studies will be required to determine whether COX-2 inhibitors prevent an increase in astroglia-derived VEGF in response to CCM endothelium. Collectively, our data are consistent with the notion that reciprocal communication between CCM endothelium and astrocytes drives CCM lesion formation and contributes to neurovascular dysfunction. These observations point to the possibility of designing therapeutic approaches aimed at preventing endothelial dysfunction and astroglia activation as an intervention to reduce the burden of CCM disease in humans.

\section{Methods}

Human tissue and genetically modified mice. Human brain samples of patients with CCM and controls without neurological disease were obtained from the Angioma Alliance Tissue Bank. Endothelial-specific conditional $P d c d 10$-null mice were generated by crossing a $P d g f b$ promoter-driven tamoxifen-regulated Cre recombinase (iCreERT2) (98) with loxP-flanked $P d c d 10$ ( $P d c d 10^{f / f l}$, a gift from Wang Min, Yale University; Pdgfb-iCreERT2 Pdcd10 $\left.{ }^{f / f l}\right)$ mice. Brain endothelialspecific conditional $P d c d 10$-null mice were generated by crossing a Slco1c1 promoter-driven tamoxifen-regulated Cre recombinase (67) (iCreERT2, a gift from Markus Schwaninger, University of Lübeck) with $P d c d 1 O^{f / f l}$ mice. eNOS-deficient mice $\left(\operatorname{Nos}^{-/-}\right)$were obtained from Jackson Laboratory and crossed with Pdgfb-iCreERT2 Pdcd10 $0^{f / f l}$ mice to generate Pdgfb-iCreERT2 Pdcd1 $O^{f / f} \mathrm{Nos}^{-/}$and Pdgfb-iCreERT2 $P d c d 10^{f / f} \mathrm{Nos}^{+/-}$mice. On postnatal day 3, mice were administered 50 $\mu \mathrm{g}$ of 4-hydroxi-tamoxifen (H7904, Sigma-Aldrich) by intragastric injection to induce genetic inactivation of the endothelial $P d c d 10$ gene in littermates with Pdgfb-iCreERT2 (Pdcd1O ${ }^{E C K O}$ ), and $P d c d 1 O^{f / f l}$ mice were used as littermate controls. For CCM in juvenile animals, on postnatal day 6 , mice were administered $100 \mu$ g 4-hydroxi-tamoxifen by intragastric injection to induce genetic inactivation of the endothelial Pdcd1O gene in littermates with Pdgfb-iCreERT2 (Pdcd10 $\left.{ }^{E C K O}\right)$, and $P d c d 10^{f / f l}$ mice were used as littermate controls. On postnatal day 1 , mice were administered $50 \mu \mathrm{g}$ tamoxifen by intragastric injection to induce genetic inactivation of endothelial $P d c d 10$ gene in littermates

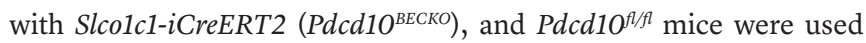
as littermate controls. Vegfa $a^{\text {tml.1Nagy }}$ mice, expressing a B-galactosidase (LacZ) reporter gene inserted into the 3 ' untranslated region of the Vegfa gene (53), were obtained from the Jackson Laboratory. The GFAP-TK mice line was used to selectively ablate proliferative astrocytes (glial fibrillary acidic protein-thymidine kinase, a gift from Michael V. Sofroniew, University of California, Los Angeles) (50, 51).

For celecoxib treatment, brain endothelial-specific conditional Pdcd10-null mice were randomized (by flipping a coin) to celecoxib (40 mg/kg, celebrex) or vehicle treatment (0.5\% methylcellulose plus $0.025 \%$ Tween20). For neonatal experiments, $100 \mu \mathrm{L}$ celecoxib or vehicle was administered by intragastric injection on P6, P7, P8, and $\mathrm{P} 9$, and animals were sacrifice at P13. For adult experiments, male and female brain endothelial-specific conditional $P d c d 10$-null mice were randomized (by flipping a coin) to celecoxib (40 mg/kg, Celebrex) or vehicle treatment $(0.5 \%$ methylcellulose plus $0.025 \%$ tween 20$)$. Celecoxib or vehicle was administered by oral gavage administration for 15 consecutive days (P55-P70) and animals were sacrificed at P80.

Western blot analysis. BMECs were lysed using a solution containing $1 \mathrm{mM}$ sodium orthovanadate, protease inhibitor cocktail (11836170001, Roche), and $100 \mu \mathrm{L}$ radioimmunoprecipitation assay buffer (Thermo Fisher Scientific). A Micro BCA protein assay kit (5000116, Thermo Fisher Scientific) was used to determine the protein concentration and $25 \mu \mathrm{g}$ cell lysates was heated for 5 minutes at $95^{\circ} \mathrm{C}$ to denature all proteins. The cell lysates were subjected to a $4 \%$ to $20 \%$ gradient SDS-PAGE (XP04200, Thermo Fisher Scientific) and a wet transfer was used to transfer proteins to nitrocellulose membranes 
(Amersham). Membranes were blocked for 1 hour at room temperature using a blocking solution (TBS 1×, 10\% nonfat milk), and polyclonal rabbit antibodies directed against eNOS (1:500; PA1-037, Thermo Fisher Scientific), HIF-1 $\alpha$ (1:150; NB100-134, Novus Biologicals) or monoclonal rabbit (1:100; 12282, Cell Signaling) and polyclonal goat (1:100; PA1-9032, Thermo Fisher Scientific) antibodies directed against COX-2 were incubated at $4^{\circ} \mathrm{C}$ overnight. Several washes were performed and then the membranes were incubated with appropriate IRDye/Alexa Fluor-coupled secondary antibodies (1:10,000, 92668070 ; 926-32211; Li-COR) for 1 hour at room temperature. A mouse monoclonal antibody against beta-actin (1:5000; A5441, SigmaAldrich) was used as a control for protein loading. Membranes were imaged and analyzed using Odyssey CLx Infrared Imaging (Li-COR).

$R T^{2}-q P C R$ analysis. Total RNA (10 ng) was used to produce single-stranded complementary DNA (cDNA) using random primers according to the manufacturer's protocol (48190011; 18080093; Thermo Fisher Scientific). Briefly, 10 ng RNA was added to a master mix containing $1 \mathrm{X}$ First-Strand buffer, $1 \mu \mathrm{L}$ 0.1M DTT, $1 \mathrm{mM}$ dNTPs, $1 \mu \mathrm{L}$ RNaseOUT recombinant RNase inhibitor, SuperScript III reverse transcriptase, and $0.5 \mu \mathrm{g}$ random primers. The mixture was placed in a thermal cycler ( $\mathrm{C} 1000$ Touch, Bio-Rad) at $25^{\circ} \mathrm{C}$ for 10 minutes, and then incubated at $50^{\circ} \mathrm{C}$ for 1 hour followed by inactivation of the reaction by heating at $70^{\circ} \mathrm{C}$ for 15 minutes. cDNA (300 ng) was run with the Kapa SybrFast qPCR mix (Kapa Biosystems) using $50 \mu \mathrm{M}$ of primers to distinguish between the relative levels of genes in each condition. The PCR reaction was placed in a thermal cycler (C1000 Touch, Bio-Rad) using an initial step at $95^{\circ} \mathrm{C}$ for 15 minutes, followed by 40 cycles (30 seconds at $95^{\circ} \mathrm{C}, 30$ seconds at $55^{\circ} \mathrm{C}$, and 30 seconds at $72^{\circ} \mathrm{C}$ ). Analysis of the data was performed using the $2^{-\Delta \mathrm{CT}}$ method.

NO determination. $P d c d 1 O^{E C K O}$ and $P d c d 1 O^{f / f l}$ BMECs were maintained in BMEC media supplemented with $500 \mu \mathrm{M} \mathrm{L}$-arginine and deficient in fetal bovine serum and ascorbic acid for 36 hours. Then, the BMEC media were collected, and a colorimetric total NO assay was performed as specified by manufacturer's protocol (KGE001, R\&D Systems). A quantity of $50 \mu \mathrm{L}$ undiluted BMEC media samples and nitrate $\left(\mathrm{NO}_{3}\right)$ standards ranging $100 \mu \mathrm{M}$ to $1.565 \mu \mathrm{M}$ were plated on clear polystyrene microplates (DY990, R\&D Systems). To measure total nitrites $\left(\mathrm{NO}_{2}\right)$ and nitrates, $25 \mu \mathrm{L}$ nitrate reductase and 25 $\mu \mathrm{L}$ NADH were added to each well to convert all available nitrates to nitrite. The plate was covered with an adhesive strip and incubated at $37^{\circ} \mathrm{C}$ for 30 minutes. After incubation, $50 \mu \mathrm{L}$ Griess reagent I and Griess reagent II were added to all wells and incubated at room temperature for 10 minutes. A 2-step diazotization reaction occurs in which $\mathrm{NO}_{2}^{-}$reacts with sufanilic acid to produce a diazonium ion, which is then coupled to N-(1-naphthyl) ethylenediamine to form an azo-derivative that absorbs light at $540 \mathrm{~nm}$. The optical density (OD) of each well was read using a microplate reader (Infinite 200 PRO,
Tecan) at $540 \mathrm{~nm}$ with a wavelength correction of $690 \mathrm{~nm}$. Duplicate readings were averaged and normalized to the blank. A standard curve was generated for each experiment by plotting optical density against concentration $(\mu \mathrm{M})$, and total nitrate/nitrite concentrations were determined using the linear trendline.

Statistics. Statistical analysis for single comparisons was performed using a 2-tailed Student's $t$ test, and analysis for multiple comparisons was performed using ANOVA followed by Tukey's post hoc test. The threshold for statistical significance was set at $P$ equal to 0.05 .

Study approval. All animal experiments were performed in compliance with animal procedure protocols approved by the University of California, San Diego Institutional Animal Care and Use Committee.

\section{Author contributions}

CCL, SIS, PH, and MALR performed and analyzed histology and gene expression experiments in culture and conditional knockout mice. CCL, SIS, and PH made substantial contributions to manuscript preparation, writing, analysis of the data, and generation of all figures. AP, RV, EJE, and SM performed histology and treatment of conditional knockout mice. RG, TM, RL, NH, RS, and IAA performed, analyzed, and interpreted the microCT studies and edited the manuscript. SM, OP, GGH, and RD contributed to data discussion and manuscript editing. BG, HS, FL, and MHG made substantial contributions to manuscript preparation, writing, and interpretation of data. MALR designed the experiments, prepared the figures, and with MHG performed overall study design to address the conceptual ideas, analysis and interpretation of the data, and writing of the manuscript.

\section{Acknowledgments}

The authors thank Angioma Alliance for providing human CCM specimens; Chelsea Hyun Ju Choi, Alyssa Castillo, Daniel Han, and Maryum Haidari for technical assistance; Jennifer Santini and Marcy Erb for microscopy technical assistance; and the UCSD School of Medicine Microscopy Core. This work was supported by National Institutes of Health, National Heart, Lung, and Blood Institute grants K01 HL133530, P01HL151433-01 (to MALR), HL139947 (to MHG), and National Institute of Neurological Disorders and Stroke grant P01 NS092521 (to MHG, IAA, RG, RL, $\mathrm{NH}$, RS, and TM). This work was also supported by Be Brave for Life Foundation (to MALR), the Future Faculty of Cardiovascular Sciences (FOCUS) PRIDE program (to MALR), as well as by the UCSD School of Medicine Microscopy Core (P30 NS047101).

Address correspondence to: Miguel Alejandro Lopez-Ramirez, 9500 Gilman Diver, BSB 5096, La Jolla, California 92093, USA. Phone: 858.534.4425; Email: malopezramirez@health.ucsd.edu.
1. Spiegler S, et al. Cerebral cavernous malformations: an update on prevalence, molecular genetic analyses, and genetic counselling. $\mathrm{Mol}$ Syndromol. 2018;9(2):60-69.

2. Fischer A, et al. Cerebral cavernous malformations: from CCM genes to endothelial cell homeostasis. Trends Mol Med. 2013;19(5):302-308.

3. Leblanc GG, et al. Biology of vascular malformations of the brain. Stroke. 2009;40(12):e694-e702.

4. Gore AV, et al. Combinatorial interaction between
CCM pathway genes precipitates hemorrhagic stroke. Dis Model Mech. 2008;1(4-5):275-281.

5. Choquet H, et al. Genetics of cerebral cavernous malformations: current status and future prospects. J Neurosurg Sci. 2015;59(3):211-220.

6. Lopez-Ramirez MA, et al. Thrombospondin1 (TSP1) replacement prevents cerebral cavernous malformations. J Exp Med. 2017;214(11):3331-3346.

7. Denier C, et al. Genotype-phenotype correlations in cerebral cavernous malformations patients.
Ann Neurol. 2006;60(5):550-556.

8. Mikati AG, et al. Vascular permeability in cerebral cavernous malformations. J Cereb Blood Flow Metab. 2015;35(10):1632-1639.

9. Lant $\mathrm{B}$, et al. Interrogating the $\mathrm{ccm}-3$ gene network. Cell Rep. 2018;24(11):2857-2868.

10. Boulday G, et al. Developmental timing of CCM2 loss influences cerebral cavernous malformations in mice. J Exp Med. 2011;208(9):1835-1847.

11. Zhou HJ, et al. Erratum: endothelial exocytosis of 
angiopoietin-2 resulting from CCM3 deficiency contributes to cerebral cavernous malformation. Nat Med. 2016;22(12):1502.

12. Chan AC, et al. Mutations in 2 distinct genetic pathways result in cerebral cavernous malformations in mice. J Clin Invest. 2011;121(5):1871-1881.

13. Zhou Z, et al. Corrigendum: cerebral cavernous malformations arise from endothelial gain of MEKK3-KLF2/4 signalling. Nature. 2016;536(7617):488.

14. McDonald DA, et al. A novel mouse model of cerebral cavernous malformations based on the two-hit mutation hypothesis recapitulates the human disease. Hum Mol Genet. 2011;20(2):211-222.

15. Akers AL, et al. Biallelic somatic and germline mutations in cerebral cavernous malformations (CCMs): evidence for a two-hit mechanism of CCM pathogenesis. Hum Mol Genet. 2009;18(5):919-930.

16. Detter MR, et al. Cerebral cavernous malformations develop through clonal expansion of mutant endothelial cells. Circ Res. 2018;123(10):1143-1151.

17. Malinverno M, et al. Endothelial cell clonal expansion in the development of cerebral cavernous malformations. Nat Commun. 2019;10(1):2761.

18. Chapman EM, et al. A conserved CCM complex promotes apoptosis non-autonomously by regulating zinc homeostasis. Nat Commun. 2019;10(1):1791.

19. Hong CC, et al. Cerebral cavernous malformations are driven by ADAMTS5 proteolysis of versican. JExp Med. 2020;217(10):e20200140.

20. Louvi A, et al. Loss of cerebral cavernous malformation $3(\mathrm{Ccm} 3)$ in neuroglia leads to CCM and vascular pathology. Proc Natl Acad Sci US A. 2011;108(9):3737-3742.

21. Finetti F, et al. KRIT1 loss-mediated upregulation of NOX1 in stromal cells promotes paracrine pro-angiogenic responses. Cell Signal. 2020;68:109527.

22. Choquet $\mathrm{H}$, et al. Cytochrome $\mathrm{P} 450$ and matrix metalloproteinase genetic modifiers of disease severity in cerebral cavernous malformation type 1. Free Radic Biol Med. 2016;92:100-109.

23. Tanriover G, et al. PDCD10, the gene mutated in cerebral cavernous malformation 3, is expressed in the neurovascular unit. Neurosurgery. 2008;62(4):930-938.

24. Daneman R, Prat A. The blood-brain barrier. Cold Spring Harb Perspect Biol. 2015;7(1):a020412.

25 . Weiss $\mathrm{N}$, et al. The blood-brain barrier in brain homeostasis and neurological diseases. Biochim Biophys Acta. 2009;1788(4):842-857.

26. Alvarez JI, et al. The Hedgehog pathway promotes blood-brain barrier integrity and CNS immune quiescence. Science. 2011;334(6063):1727-1731.

27. Liddelow SA, et al. Neurotoxic reactive astrocytes are induced by activated microglia. Nature. 2017;541(7638):481-487.

28. Hamby ME, Sofroniew MV. Reactive astrocytes as therapeutic targets for CNS disorders. Neurotherapeutics. 2010;7(4):494-506.

29. Argaw AT, et al. Astrocyte-derived VEGF-A drives blood-brain barrier disruption in CNS inflammatory disease. J Clin Invest.
2012;122(7):2454-2468.

30. Vangeison G, Rempe DA. The Janus-faced effects of hypoxia on astrocyte function. Neuroscientist. 2009;15(6):579-588.

31. Chapouly C, et al. Astrocytic TYMP and VEGFA drive blood-brain barrier opening in inflammatory central nervous system lesions. Brain. 2015;138(Pt 6):1548-1567.

32. Maiuri F, et al. Clinical progression and familial occurrence of cerebral cavernous angiomas: the role of angiogenic and growth factors. Neurosurg Focus. 2006;21(1):e3.

33. Jung KH, et al. Cerebral cavernous malformations with dynamic and progressive course: correlation study with vascular endothelial growth factor. Arch Neurol. 2003;60(11):1613-1618.

34. Abe T, et al. The association between high VEGF levels and multiple probable punctuate cavernous malformations. Acta Neurochir (Wien). 2009;151(7):855-859.

35. Girard R, et al. Plasma biomarkers of inflammation and angiogenesis predict cerebral cavernous malformation symptomatic hemorrhage or lesional growth. Circ Res. 2018;122(12):1716-1721.

36. Argaw AT, et al. VEGF-mediated disruption of endothelial CLN-5 promotes blood-brain barrier breakdown. Proc Natl Acad Sci U S A. 2009;106(6):1977-1982.

37. Cheng SY, et al. Intracerebral tumor-associated hemorrhage caused by overexpression of the vascular endothelial growth factor isoforms VEGF121 and VEGF165 but not VEGF189. Proc Natl Acad Sci U S A. 1997;94(22):12081-12087.

38. Josko J. Cerebral angiogenesis and expression of VEGF after subarachnoid hemorrhage (SAH) in rats. Brain Res. 2003;981(1-2):58-69.

39. Awad IA, Polster SP. Cavernous angiomas: deconstructing a neurosurgical disease. J Neurosurg. 2019;131(1):1-13.

40. Lopez-Ramirez MA, et al. Cerebral cavernous malformations form an anticoagulant vascular domain in humans and mice. Blood. 2019;133(3):193-204.

41. DiStefano PV, et al. KRIT1 protein depletion modifies endothelial cell behavior via increased vascular endothelial growth factor (VEGF) signaling. J Biol Chem. 2014;289(47):33054-33065.

42. Dimmeler S, et al. Activation of nitric oxide synthase in endothelial cells by Akt-dependent phosphorylation. Nature. 1999;399(6736):601-605.

43. Fulton D, et al. Regulation of endothelium-derived nitric oxide production by the protein kinase Akt. Nature. 1999;399(6736):597-601.

44. Fukumura D, et al. Predominant role of endothelial nitric oxide synthase in vascular endothelial growth factor-induced angiogenesis and vascular permeability. Proc Natl Acad Sci U S A. 2001;98(5):2604-2609.

45. Jozkowicz A, et al. Genetic augmentation of nitric oxide synthase increases the vascular generation of VEGF. Cardiovasc Res. 2001;51(4):773-783.

46. Zhang R, et al. Nitric oxide enhances angiogenesis via the synthesis of vascular endothelial growth factor and cGMP after stroke in the rat. Circ Res. 2003;92(3):308-313.

47. Cuttano R, et al. KLF4 is a key determinant in the development and progression of cerebral cavernous malformations. EMBOMolMed. 2016;8(1):6-24.
48. Renz M, et al. Regulation of $\beta 1$ integrin-Klf2-mediated angiogenesis by CCM proteins. Dev Cell. 2015;32(2):181-190.

49. Leto K, et al. Consensus paper: cerebellar development. Cerebellum. 2016;15(6):789-828.

50. Faulkner JR, et al. Reactive astrocytes protect tissue and preserve function after spinal cord injury. J Neurosci. 2004;24(9):2143-2155.

51. Anderson MA, et al. Astrocyte scar formation aids central nervous system axon regeneration. Nature. 2016;532(7598):195-200.

52. Girard R, et al. Micro-computed tomography in murine models of cerebral cavernous malformations as a paradigm for brain disease. JNeurosci Methods. 2016;271:14-24.

53. Miquerol L, et al. Multiple developmental roles of VEGF suggested by a LacZ-tagged allele. Dev Biol. 1999;212(2):307-322.

54. Scott A, et al. Astrocyte-derived vascular endothelial growth factor stabilizes vessels in the developing retinal vasculature. PLoS One. 2010;5(7):e11863.

55. Cardoso C, et al. Novel chronic mouse model of cerebral cavernous malformations. Stroke. 2020;51(4):1272-1278.

56. Detter MR, et al. Novel murine models of cerebral cavernous malformations. Angiogenesis. 2020;23(4):651-666.

57. Li W, et al. Propranolol inhibits cavernous vascular malformations by $\beta 1$ adrenergic receptor antagonism in animal models. J Clin Invest. 2021;131(3):144893.

58. Detter MR, et al. Novel hemorrhage models of cerebral cavernous malformations [preprint]. https://doi.org/10.1101/2020.02.12.944421. Posted on bioRxiv February 13, 2020.

59. Majmundar AJ, et al. Hypoxia-inducible factors and the response to hypoxic stress. Mol Cell. 2010;40(2):294-309.

60. Semenza GL. Hypoxia-inducible factor 1: oxygen homeostasis and disease pathophysiology. Trends Mol Med. 2001;7(8):345-350.

61. Benita $Y$, et al. An integrative genomics approach identifies hypoxia inducible factor-1 (HIF-1)-target genes that form the core response to hypoxia. Nucleic Acids Res. 2009;37(14):4587-4602.

62. Kaidi A, et al. Direct transcriptional up-regulation of cyclooxygenase- 2 by hypoxia-inducible factor (HIF)-1 promotes colorectal tumor cell survival and enhances HIF-1 transcriptional activity during hypoxia. Cancer Res. 2006;66(13):6683-6691.

63. Simon MC. The hypoxia response pathways hats off! N Engl JMed. 2016;375(17):1687-1689.

64. Koskimaki J, et al. Comprehensive transcriptome analysis of cerebral cavernous malformation across multiple species and genotypes. JCI Insight. 2019;4(3):e126167.

65. Hamik A, et al. Kruppel-like factor 4 regulates endothelial inflammation. J Biol Chem. 2007;282(18):13769-13779.

66. SenBanerjee S, et al. KLF2 Is a novel transcriptional regulator of endothelial proinflammatory activation. J Exp Med. 2004;199(10):1305-1315.

67. Ridder DA, et al. TAK1 in brain endothelial cells mediates fever and lethargy. J Exp Med. 2011;208(13):2615-2623.

68. Abe M, et al. Thrombus and encapsulated hematoma in cerebral cavernous malformations. Acta 
Neuropathol. 2005;109(5):503-509.

69. Bardehle S, et al. Live imaging of astrocyte responses to acute injury reveals selective juxtavascular proliferation. Nat Neurosci. 2013;16(5):580-586.

70. Anderson MA, et al. Heterogeneity of reactive astrocytes. Neurosci Lett. 2014;565:23-29.

71. Rothhammer V, et al. Microglial control of astrocytes in response to microbial metabolites. Nature. 2018;557(7707):724-728.

72. Cirino G, et al. Endothelial nitric oxide synthase: the Cinderella of inflammation? Trends Pharmacol Sci. 2003;24(2):91-95.

73. Lin S, et al. Sustained endothelial nitric-oxide synthase activation requires capacitative $\mathrm{Ca} 2+$ entry. J Biol Chem. 2000;275(24):17979-17985.

74. Kimura H, Esumi H. Reciprocal regulation between nitric oxide and vascular endothelial growth factor in angiogenesis. Acta Biochim Pol. 2003;50(1):49-59.

75. Schulz GB, et al. Cerebral cavernous malformation-1 protein controls DLL4-Notch3 signaling between the endothelium and pericytes. Stroke. 2015;46(5):1337-1343.

76. Stamatovic SM, et al. PDCD10 (CCM3) regulates brain endothelial barrier integrity in cerebral cavernous malformation type 3: role of CCM3-ERK1/2-cortactin cross-talk. Acta Neuropathol. 2015;130(5):731-750.

77. Engelhardt S, et al. Cell-specific blood-brain barrier regulation in health and disease: a focus on hypoxia. Br J Pharmacol. 2014;171(5):1210-1230.

78. Shi Q, et al. Nitric oxide from brain microvascular endothelial cells may initiate the compensatory response to mild hypoxia of astrocytes in a hypoxia-inducible factor-1 $\alpha$ dependent manner. Am J Transl Res. 2016;8(11):4735-4749.
79. Brix B, et al. Endothelial cell-derived nitric oxide enhances aerobic glycolysis in astrocytes via HIF-1 $\alpha$-mediated target gene activation. J Neurosci. 2012;32(28):9727-9735.

80. Atochin DN, Huang PL. Endothelial nitric oxide synthase transgenic models of endothelial dysfunction. Pflugers Arch. 2010;460(6):965-974.

81. Huang PL. Endothelial nitric oxide synthase and endothelial dysfunction. Curr Hypertens Rep. 2003;5(6):473-480.

82. Li Q, et al. Diabetic eNOS-knockout mice develop accelerated retinopathy. Invest Ophthalmol Vis Sci. 2010;51(10):5240-5246.

83. Nakagawa $\mathrm{T}$, et al. Diabetic endothelial nitric oxide synthase knockout mice develop advanced diabetic nephropathy. J Am Soc Nephrol. 2007;18(2):539-550.

84. Opatrilova R, et al. Nitric oxide in the pathophysiology of retinopathy: evidences from preclinical and clinical researches. Acta Ophthalmol. 2018;96(3):222-231.

85. Metzen E, et al. Nitric oxide impairs normoxic degradation of HIF-1alpha by inhibition of prolyl hydroxylases. Mol Biol Cell. 2003;14(8):3470-3481.

86. Palmer LA, et al. Normoxic stabilization of hypoxia-inducible factor-1 expression and activity: redox-dependent effect of nitrogen oxides. Mol Pharmacol. 2000;58(6):1197-1203.

87. Schleicher M, et al. The Akt1-eNOS axis illustrates the specificity of kinase-substrate relationships in vivo. Sci Signal. 2009;2(82):ra41.

88. Chakraborty A, et al. Angiopoietin like- 4 as a novel vascular mediator in capillary cerebral amyloid angiopathy. Brain. 2018;141(12):3377-3388.

89. Katanasaka Y, et al. Epidermal growth factor receptor variant type III markedly accelerates angiogenesis and tumor growth via inducing c-myc mediated angiopoietin-like 4 expression in malignant glioma. Mol Cancer. 2013;12:31.

90. Iniguez MA, et al. Cyclooxygenase-2: a therapeutic target in angiogenesis. Trends Mol Med. 2003;9(2):73-78.

91. Gately S, Li WW. Multiple roles of COX-2 in tumor angiogenesis: a target for antiangiogenic therapy. Semin Oncol. 2004;31(2 Suppl 7):2-11.

92. Flemming KD, et al. Predictors of initial presentation with hemorrhage in patients with cavernous malformations. World Neurosurg. 2020;133:e767-e773.

93. Polster SP, et al. Trial readiness in cavernous angiomas with symptomatic hemorrhage (CASH). Neurosurgery. 2019;84(4):954-964.

94. Jones MK, et al. Inhibition of angiogenesis by nonsteroidal anti-inflammatory drugs: insight into mechanisms and implications for cancer growth and ulcer healing. Nat Med.1999;5(12):1418-1423.

95. Majima M, et al. Cyclo-oxygenase-2 enhances basic fibroblast growth factor-induced angiogenesis through induction of vascular endothelial growth factor in rat sponge implants. Br JPharmacol. 2000;130(3):641-649.

96. Xu L, et al. COX-2 inhibition potentiates antiangiogenic cancer therapy and prevents metastasis in preclinical models. Sci Transl Med. 2014;6(242):242ra84.

97. Bravi L, et al. Sulindac metabolites decrease cerebrovascular malformations in CCM3-knockout mice. Proc Natl Acad Sci U S A. 2015;112(27):8421-8426.

98. Claxton S, et al. Efficient, inducible Cre-recombinase activation in vascular endothelium. Genesis. 2008;46(2):74-80. 